Journal for ImmunoTherapy of Cancer

\title{
Neoantigen vaccination induces clinical and immunologic responses in non- small cell lung cancer patients harboring EGFR mutations
}

Fenge Li (D) , ${ }^{1}$ Ligang Deng, ${ }^{2}$ Kyle R Jackson, ${ }^{1}$ Amjad H Talukder, ${ }^{1}$ Arjun S Katailiha, ${ }^{1}$ Sherille D Bradley, ${ }^{1}$ Qingwei Zou, ${ }^{2}$ Caixia Chen, ${ }^{2}$ Chong Huo, ${ }^{2}$ Yulun Chiu, ${ }^{1}$ Matthew Stair, ${ }^{3}$ Weihong Feng, ${ }^{4}$ Aleksander Bagaev, ${ }^{5}$ Nikita Kotlov, ${ }^{5}$ Viktor Svekolkin, ${ }^{5}$ Ravshan Ataullakhanov, ${ }^{5}$ Natalia Miheecheva, ${ }^{5}$ Felix Frenkel, ${ }^{5}$ Yaling Wang, ${ }^{2}$ Minying Zhang, ${ }^{1}$ David Hawke, ${ }^{6}$ Ling Han, ${ }^{4}$ Shuo Zhou, ${ }^{7}$ Yan Zhang, ${ }^{8}$ Zhenglu Wang, ${ }^{9}$ William K Decker, ${ }^{10}$ Heather M Sonnemann, ${ }^{1}$ Jason Roszik, ${ }^{1}$ Marie-Andree Forget, ${ }^{1}$ Michael A Davies (D) , ${ }^{1}$ Chantale Bernatchez (D) ,1 Cassian Yee, ${ }^{1,11}$ Roland Bassett, ${ }^{12}$ Patrick Hwu (D) , ${ }^{1}$ Xueming Du, ${ }^{4}$ Gregory Lizee (D) 1,11

To cite: Li F, Deng L, Jackson KR, et al. Neoantigen vaccination induces clinical and immunologic responses in nonsmall cell lung cancer patients harboring EGFR mutations. Journal for ImmunoTherapy of Cancer 2021;9:e002531. doi:10.1136/jitc-2021-002531

- Additional supplemental material is published online only. To view, please visit the journal online (http://dx.doi.org/10. 1136/jitc-2021-002531).

Accepted 07 June 2021

\section{Check for updates}

(c) Author(s) (or their employer(s)) 2021. Re-use permitted under CC BY-NC. No commercial re-use. See rights and permissions. Published by BMJ.

For numbered affiliations see end of article.

\section{Correspondence to} Dr Gregory Lizee; glizee@mdanderson.org

Dr Xueming Du; dudaming73@163.com

\section{ABSTRACT}

Background Neoantigen (NeoAg) peptides displayed at the tumor cell surface by human leukocyte antigen molecules show exquisite tumor specificity and can elicit T cell mediated tumor rejection. However, few NeoAgs are predicted to be shared between patients, and none to date have demonstrated therapeutic value in the context of vaccination.

Methods We report here a phase I trial of personalized NeoAg peptide vaccination (PPV) of 24 stage III/IV non-small cell lung cancer (NSCLC) patients who had previously progressed following multiple conventional therapies, including surgery, radiation, chemotherapy, and tyrosine kinase inhibitors (TKIs). Primary endpoints of the trial evaluated feasibility, tolerability, and safety of the personalized vaccination approach, and secondary trial endpoints assessed tumor-specific immune reactivity and clinical responses. Of the 16 patients with epidermal growth factor receptor (EGFR) mutations, nine continued TKI therapy concurrent with PPV and seven patients received PPV alone.

Results Out of 29 patients enrolled in the trial, 24 were immunized with personalized NeoAg peptides. Aside from transient rash, fatigue and/or fever observed in three patients, no other treatment-related adverse events were observed. Median progression-free survival and overall survival of the 24 vaccinated patients were 6.0 and 8.9 months, respectively. Within 3-4 months following initiation of PPV, seven RECIST-based objective clinical responses including one complete response were observed. Notably, all seven clinical responders had EGFR-mutated tumors, including four patients that had continued TKI therapy concurrently with PPV. Immune monitoring showed that five of the seven responding patients demonstrated vaccine-induced $T$ cell responses against EGFR NeoAg peptides. Furthermore, two highly shared EGFR mutations (L858R and T790M) were shown to be immunogenic in four of the responding patients, all of whom demonstrated increases in peripheral blood neoantigen-specific CD8+ Tcell frequencies during the course of PPV.

Conclusions These results show that personalized NeoAg vaccination is feasible and safe for advanced-stage NSCLC patients. The clinical and immune responses observed following PPV suggest that EGFR mutations constitute shared, immunogenic neoantigens with promising immunotherapeutic potential for large subsets of NSCLC patients. Furthermore, PPV with concurrent EGFR inhibitor therapy was well tolerated and may have contributed to the induction of PPV-induced T cell responses.

\section{INTRODUCTION}

Non-small-cell lung cancer (NSCLC) accounts for $\sim 85 \%$ of all lung cancer cases and is often diagnosed at late stages, resulting in a poor prognosis and only a 15\%-20\% 5-year survival rate with approved standard treatments. ${ }^{12}$ Epidermal growth factor receptor (EGFR) inhibitor (EGFRi) therapies have been highly effective at inducing tumor regressions in EGFR-mutated NSCLC, and the third-generation EGFRi osimertinib has now been approved as a first-line treatment. However, although nearly all patients initially respond to EGFRi, most experience disease recurrence within 1-2 years due to acquired drug resistance. ${ }^{3}$ The high rate of somatic mutations found in NSCLC suggests that this cancer type may be particularly amenable to immunotherapeutic interventions, a notion supported by the encouraging response rates of NSCLC patients to checkpoint blockade therapies. However, the subset of NSCLC 
patients whose tumors bear EGFR mutations respond relatively poorly to checkpoint blockade approaches, ${ }^{4-6}$ meaning that those patients who progress on EGFRi therapy currently have few if any effective treatment options.

Tumor mutation-encoding neoantigen (NeoAg) peptides presented by cell surface major histocompatibility complex (MHC) proteins can constitute targets of tumor-specific T cell mediated immunity. ${ }^{7-11} \mathrm{NeoAg}$ vaccination has demonstrated antitumor efficacy in several preclinical models, and recent clinical trials employing RNA-based or peptide-based personalized NeoAg vaccine approaches have successfully induced NeoAg-specific $\mathrm{T}$ cells associated with clinical responses in melanoma patients treated concurrently with checkpoint blockade therapy. ${ }^{12-17}$ Furthermore, recent personalized peptide vaccine trials for glioblastoma patients were shown to be successful at inducing NeoAg-specific $\mathrm{CD}^{+}$and $\mathrm{CD}^{+} \mathrm{T}$ cell responses associated with increased intratumoral $\mathrm{T}$ cell infiltration, though no clinical responses were reported. ${ }^{1819}$

We report here the results of a phase I clinical trial of personalized NeoAg peptide vaccination (PPV) for advanced-stage NSCLC patients who had previously progressed on multiple standard therapies including EGFRi. Aside from a transient rash, fatigue and/or fever reported in three patients, no other treatment-related adverse events were observed. Of the 24 patients immunized, seven experienced objective clinical responses by Response Evaluation Criteria In Solid Tumors (RECIST) V.1.1 criteria, including six partial responses (PRs) and one complete response (CR). Interestingly, all seven of the responding patients had tumors bearing EGFR mutations, and five of these patients demonstrated dominant EGFR NeoAg-specific $T$ cell responses following PPV. By contrast, none of the eight patients whose tumors expressed wild-type EGFR experienced clinical responses following PPV, and these patients also generated comparatively weaker PPV-specific immune responses. Molecular and cellular analyses showed that in addition to its wellknown effects on tumor cell proliferation and survival, EGFRi impacted multiple immunomodulatory pathways within lung cancer cells that favored immune cell infiltration and tumor antigen presentation, potentially contributing to the stronger antitumor immune responses observed in these patients. Taken together, these findings demonstrate that PPV is feasible, safe, and may induce tumor regressions in subsets of NSCLC patients bearing shared and immunogenic EGFR mutations, even in the setting of prior EGFRi failure.

\section{RESULTS}

\section{Patient characteristics, PPV administration, and response assessment}

We previously reported a stage IV NSCLC patient who experienced a remarkable regression of multiple lung tumors following PPV that was associated with $\mathrm{CD} 8^{+} \mathrm{T}$ cell responses against the widely shared EGFR-L858R mutation. ${ }^{20}$ Based on this case study, we initiated a phase Ib clinical trial of PPV for stage III/IV NSCLC patients to determine the safety and feasibility of PPV, with secondary endpoints being to assess clinical response in addition to the immunogenicity of personalized and shared NeoAgs (figure 1A). Personalized NeoAg peptide vaccines were successfully designed for all 29 of the eligible patients, and 24 of these patients (18 adenocarcinoma and 6 squamous cell carcinoma) received weekly PPV immunizations followed by response evaluation by CT scans (table 1 ). Patients were vaccinated for a minimum of 12 weeks and given the option to continue PPV beyond 12 weeks; the immunization time courses of all 24 patients are shown in online supplemental figure S1. Clinical responses were assessed using RECIST V.1.1 at time points 3-4 months following the initiation of PPV. Three of the evaluable patients (patients 1, 6, and 24) received alternative salvage therapy following disease progression after 12 weeks of immunization and were taken off study. Five patients did not complete CT-based staging: three patients (patients 9,19 , and 20) expired from non-treatment related causes during the 12-week immunization period; two additional study patients (patients 13 and 15) received follow-up evaluations at outside hospitals, where CT scan reports with target tumor measurements were generated 3-4 months post-PPV and subsequently communicated to the study authors. A complete summary of patient treatments and clinical outcomes is shown in online supplemental figure S2A.

All 24 NSCLC patients had previously progressed on multiple lines of conventional therapy, including surgery, radiation therapy, and/or chemotherapy. Sixteen of the patients bearing EGFR-mutated tumors had also previously progressed on EGFRi therapy, with nine of these patients having progressed on two or more different TKI drugs. These 16 patients were given the option to either stop or continue EGFRi therapy concurrent with PPV immunization; nine of the patients chose to continue receiving EGFRi. Although patients were not initially randomized into separate cohorts, as discussed further, post hoc analyses revealed distinct clinical response profiles when the 24 patients were divided into the following three subgroups: EGFR wild-type (WT) patients receiving PPV (group 1: $\mathrm{n}=8$ ), EGFR-mutated patients receiving PPV (group 2: $\mathrm{n}=7$ ); and EGFR-mutated patients receiving PPV with concurrent EGFRi (group 3: $n=9$ ) (figure 1B,C). Major clinical characteristics of the three patient groups did not differ significantly at baseline (online supplemental figure S3A), and detailed histories of EGFRi treatments for groups 2 and 3 patients are provided (online supplemental figure S3B).

\section{Personalized neoantigen vaccine design}

Large-scale whole exome sequencing studies have demonstrated that NSCLC has one of the highest mutational burdens of all cancers, with individual patient tumors often expressing several hundreds of non-synonymous somatic mutations. ${ }^{21} 22$ Although NeoAgs represent ideal 

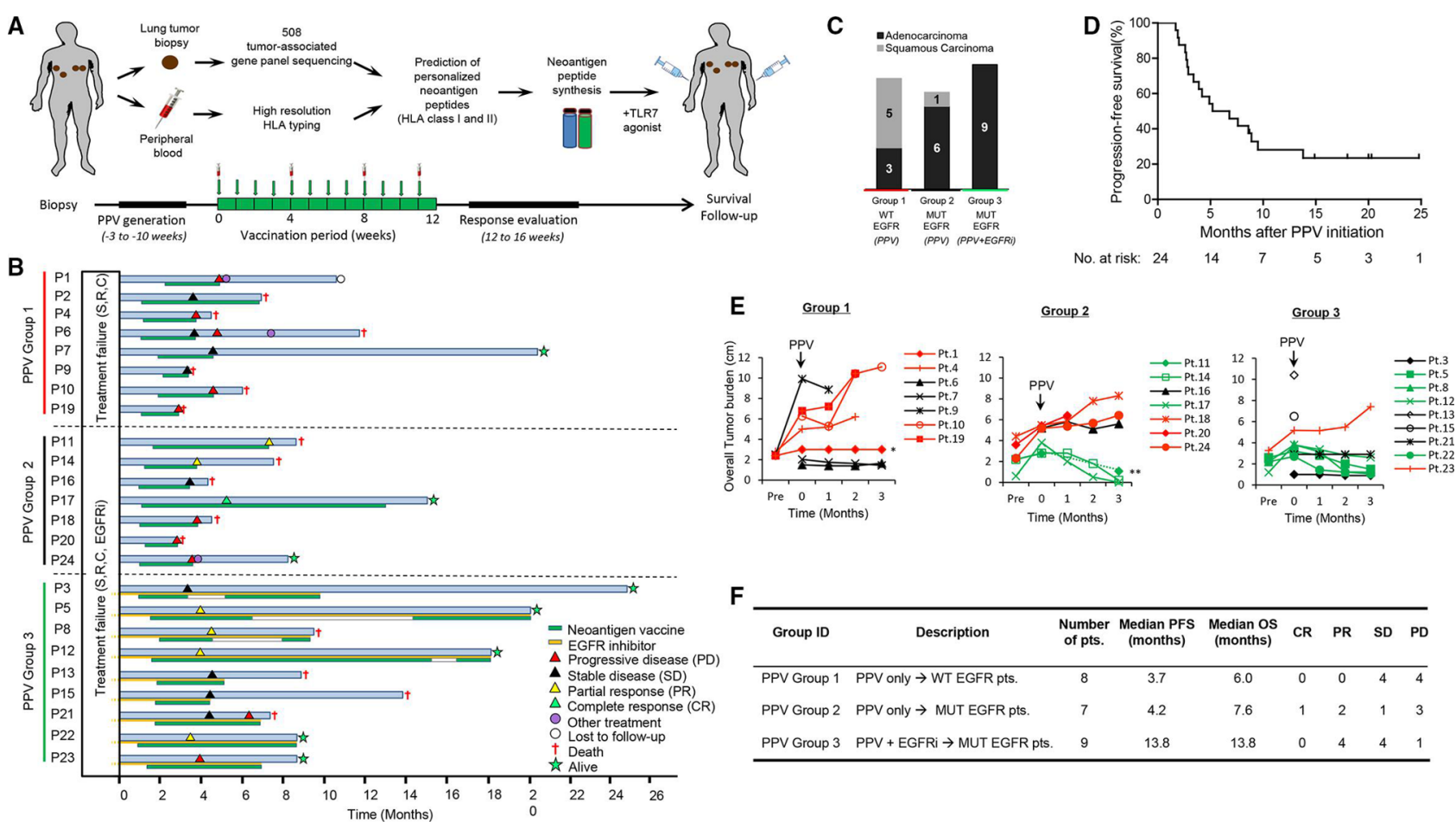

\begin{tabular}{llcccccccc}
\hline F & Description & $\begin{array}{c}\text { Number } \\
\text { of pts. }\end{array}$ & $\begin{array}{c}\text { Median PFS } \\
\text { (months) }\end{array}$ & $\begin{array}{c}\text { Median OS } \\
\text { (months) }\end{array}$ & CR & PR & SD & PD \\
\hline PPV Group 1 & PPV only $\rightarrow$ WT EGFR pts. & 8 & 3.7 & 6.0 & 0 & 0 & 4 & 4 \\
PPV Group 2 & PPV only $\rightarrow$ MUT EGFR pts. & 7 & 4.2 & 7.6 & 1 & 2 & 1 & 3 \\
PPV Group 3 & PPV + EGFRi $\rightarrow$ MUT EGFR pts. & 9 & 13.8 & 13.8 & 0 & 4 & 4 & 1 \\
\hline
\end{tabular}

Figure 1 PPV trial design and patient outcomes. (A) Neoantigen peptide vaccine manufacturing pipeline leading to immunization of patients with advanced non-small cell lung cancer (NSCLC). DNA from lung tumor biopsies was sequenced using a panel of 508 tumor-associated genes, while high-resolution HLA typing was performed on patient peripheral blood. Neoantigen vaccine peptides were selected largely based on HLA class I and class II peptide binding predictions (methods). Each patient was immunized weekly with a unique saline-based mixture of short and long neoantigen peptides divided into two cocktails and administered into opposite extremities for 12 weeks. Green arrows represent weeks when vaccination was received. Blood was drawn at weeks 0, 4, 8 and 12 as indicated with red syringe symbols. (B) Clinical event timeline for the 24 NSCLC patients who received PPV. Green bars: duration of PPV immunization. Orange bars: duration of EGFR inhibitor therapy. (C) Tumor histology of the 24 NSCLC study patients as divided into three subgroups based on EGFR mutation status and use of EGFR inhibitor during vaccination. (D) Progression-free survival curve of all 24 PPV patients. (E) Measurements of the overall tumor burden (sum of all target lesions) of PPV patients over the course of treatment. The clinical response of each patient is indicated by color: red: PD; black: SD; green: CR or PR. Tumor burden at the time point 'pre' indicates the tumor burden measured prior to patient disease progression. Tumor burden at the time point ' 0 ' indicates the tumor burden immediately prior to the start of PPV treatment. *Patient 1 developed pleural effusion at 12 weeks. ${ }^{* *}$ Follow-up CT scan of patient 11 was taken at 24 weeks. No tumor measurements are shown for patient 2 due to their having no measurable tumors. Follow-up CT scans were not available for patients 13 and 15, but other clinical follow-up information regarding response and survival was obtained. (F) Response summary of immunized patients by group showing progression-free survival, overall survival, and clinical response as assessed using RECIST V.1.1 criteria 12-18 weeks following initiation of PPV. Response of patients 9, 19 and 20 were assessed at 6-8 weeks after PPV initiation due to on-treatment death from disease progression. Survival curve was generated using Kaplan-Meier estimate. CR, complete response; EGFR, epidermal growth factor receptor; HLA, human leukocyte antigen; MUT, mutated; PD, progressive disease; PPV, personalized NeoAg peptide vaccine; PR, partial response; RECIST, Response Evaluation Criteria In Solid Tumors; SD, stable disease.

tumor-specific target antigens, the private nature of most tumor-associated mutations presents serious challenges for personalized NeoAg identification, including but not limited to: (1) lack of validation with respect to target peptide immunogenicity and/or tumor antigen presentation; (2) a lack of knowledge regarding the precise binding characteristics of most human leukocyte antigen (HLA) class I and II molecules to NeoAg peptides; (3) a predominance of passenger or 'branch' mutations not essential for tumor survival, among considerably fewer driver mutations (eg, KRAS or TP53) that more often show the 'truncal' characteristics more desirable for NeoAg targets. ${ }^{23}{ }^{24}$ Furthermore, based on mutational profiling, HLA class I peptide-binding prediction algorithms typically generate numbers of $\mathrm{NeoAg}$ peptide candidates that are orders of magnitude larger than can feasibly be incorporated into a multiepitope peptide vaccine. In order to focus on targeting shared driver mutations with our PPV approach, we chose to perform mutational profiling on a panel of 508 known cancer-associated genes using DNA from needle-biopsied fresh tumor tissue (online supplemental table S1). Vaccine peptides were chosen primarily based on the highest predicted binding affinity of mutation-encoding NeoAgs to each patient's individual HLA class I and class II allotypes (online supplemental table S2, see online supplemental methods). Each patient was immunized with a unique, personalized mixture of short and long NeoAg peptides dissolved in saline, divided into two pools and administered subcutaneously 
Table 1 Clinical baseline characteristics of study patients

\begin{tabular}{ll}
\hline Characteristic & Patients (n=24) \\
\hline Gender, $n$ (\%) & $10(41.7)$ \\
\hline Male & $14(58.3)$ \\
\hline Female & \\
Age (year) & $60.13 \pm 9.37$ \\
\hline Mean \pm SD & $45-84$ \\
\hline Range & \\
Weight (kg) & $65.61 \pm 12.48$ \\
\hline Mean $\pm S D$ & $48.5-105$ \\
\hline Range & $11(45.8)$ \\
\hline Smoke history (yes, \%) & $15(62.5)$ \\
\hline Pleural effusion (yes, \%) & \\
\hline Tumor burden (cm) & $4.55 \pm 2.38$ \\
\hline Mean $\pm S D$ & $1.0-10.4$ \\
\hline Range & $5-19$ \\
\hline Tumor number (S-M) & $16(66.7)$ \\
\hline EGFR mutation (yes, \%) & \\
\hline Clinical stage & 5 \\
\hline IIIA/B & 19 \\
\hline IV & \\
\hline
\end{tabular}

Previous treatment strategies:

$\begin{array}{ll}\text { Surgery (yes, \%) } & 4(16.7) \\ \text { Radiotherapy (yes, \%) } & 18(75.0) \\ \text { Chemotherapy (yes, \%) } & 17(70.8) \\ \text { EGFR inhibitor (yes, \%) } & 16(66.7) \\ \text { Brian metastases (yes,\%) } & 1(4.2) \\ \text { Tumor histology (SQ-AD) } & 6-18\end{array}$

$A D$, adenocarcinoma; EGFR, epidermal growth factor receptor; $M$, multiple; $\mathrm{S}$, single; $\mathrm{SQ}$, squamous cell carcinoma.

into opposite extremities. Imiquimod was applied topically after each immunization to provide costimulatory signals through Toll-like receptor 7 (TLR7) (figure 1A, online supplemental methods).

Among the 24 immunized patients, a mean of 6.1 coding mutations were detected per tumor (range: 1-20). Of the 16 patients with EGFR-mutated tumors, 14 harbored common EGFR driver mutations (seven patients with L858R point mutations and seven demonstrating Exon 19 deletions), two of which were also accompanied by the T790M mutation, a frequently acquired resistance mechanism to first-generation EGFR inhibitors. Two additional patients harbored the comparatively rare H773L EGFR mutation. The number of vaccine peptides administered ranged from 5 to 14 peptides per patient (mean: 9.4), which was primarily determined by the number of NeoAgs predicted to bind to patient HLA class I (mean: 6.5 peptides) or HLA class II (mean: 2.9 peptides). Patients in groups 2 and 3 received a mean of four mutated EGFR peptides each (mean: 2.8 short and 1.2 long). The number of immunizing peptides or mean predicted peptide binding affinity did not differ significantly between patient groups; however, patients in groups 2 and 3 received vaccines targeting significantly fewer somatic mutations overall (online supplemental figure S4A-C). This was due to the intentional targeting of individual EGFR mutations with multiple NeoAg peptides, an approach that had been successful for inducing the lung tumor regression observed in our initial case study patient (online supplemental table S2). ${ }^{20}$

\section{Neoantigen peptide vaccination reversed tumor progression in multiple patients with EGFR-mutated tumors}

Clinical outcomes for all 24 PPV patients are shown in table 2, with subgroup outcomes summarized in figure 1 . Aside from grade 1 transient rashes, fatigue and/or fever experienced by three patients, no treatment-related adverse events were observed (online supplemental figure S2B). With a median follow-up time of 7.1 months (range: 1.2-23.9 months), the median PFS and OS of the $24 \mathrm{PPV}$ patients were 6.0 and 8.9 months, respectively (figure 1D, online supplemental figure S2C). Of the eight EGFR-WT patients in group 1, post-PPV CT scans showed four patients with stable disease (SD) and four with progressive disease (PD). Although patient 2 experienced clearance of pleural fluid, no tumor regression was observed (online supplemental figure S5A). However, objective clinical responses were observed in three of the seven patients from group 2, including two PRs (patients 11 and 14) and one CR (patient 17) that was confirmed by a post-treatment biopsy showing no remaining viable tumor cells (figure 2A-D). Moreover, four of the nine patients in group 3 experienced PRs (figures 1E,F and 2E,F), despite prior disease progression on EGFRi monotherapy (online supplemental figure S5B). Notably, none of the patients in group 3 had taken a break or 'drug holiday' from EGFRi treatment prior to starting PPV, strongly suggesting that NeoAg vaccination played a key role in these clinical responses.

Though groups 2 and 3 patients demonstrated similar objective response rates to PPV $(42.9 \%$ vs $44.4 \%$, respectively, $95 \%$ CI 0.313 to 2.971), exploratory subgroup analysis showed that patients in groups 1,2 and 3 experienced progression-free survival (PFS) of 3.7, 4.2, and 13.8 months, and overall survival (OS) of 6.0, 7.6, and 13.8 months, respectively (figure 1F). Neither clinical response nor PFS were associated with the number of immunizing peptides, peptide length, predicted HLA binding affinity, the number of HLA molecules engaged, and peptide delta score (online supplemental figure S4A-F). However, PR/CR patients received vaccines targeting fewer somatic mutations overall compared with the vaccines of PD patients $(\mathrm{p}=0.014)$, with responding patients receiving a significantly higher proportion of EGFR NeoAg peptides in their vaccines $(p<0.001$, Online supplemental figure S4C). 


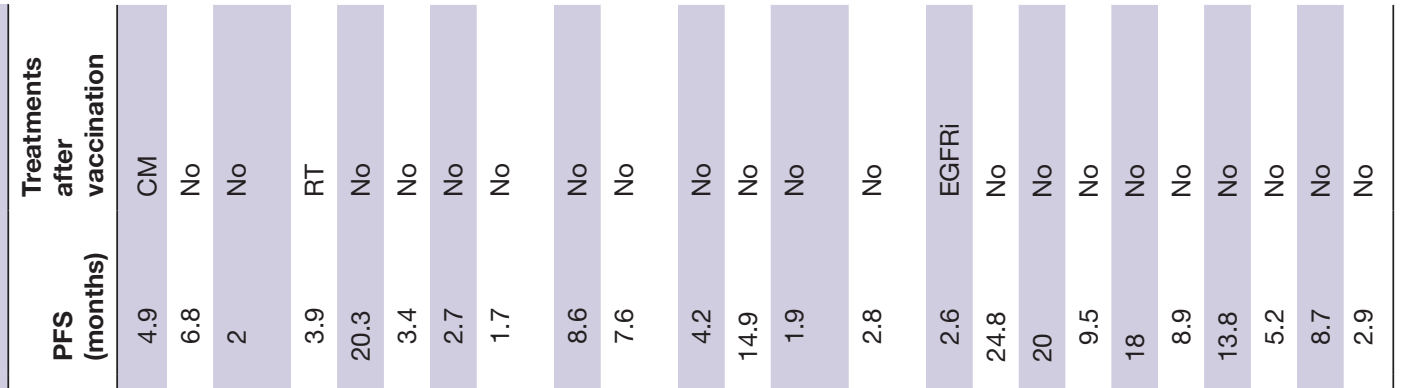

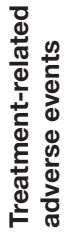

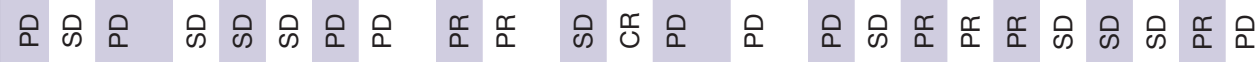

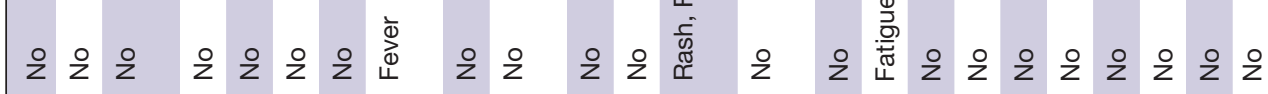
는

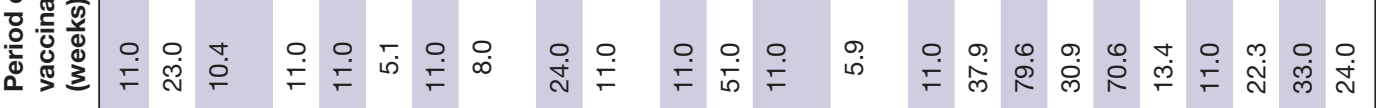

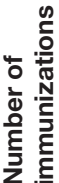

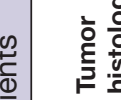

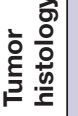

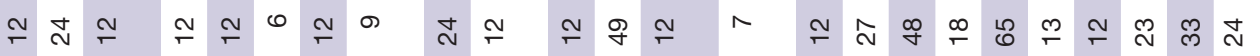

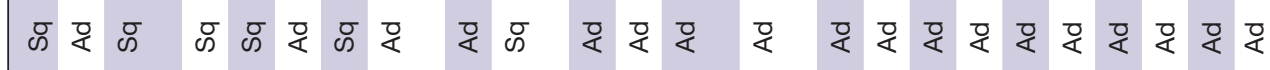
은

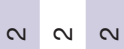

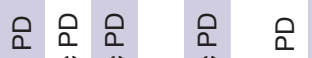
옹

$\therefore \quad \therefore \quad 0$

으음

б.

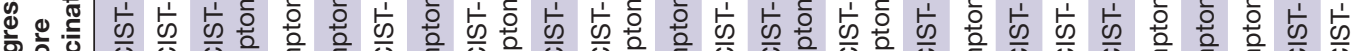
कㅇํㅇ ड

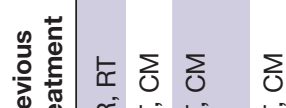

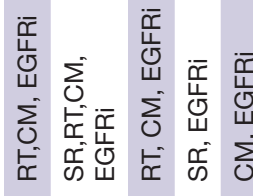

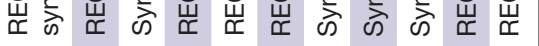

¿্ّ

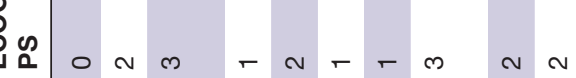

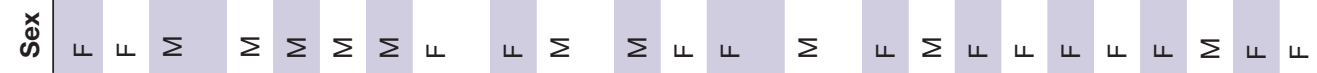

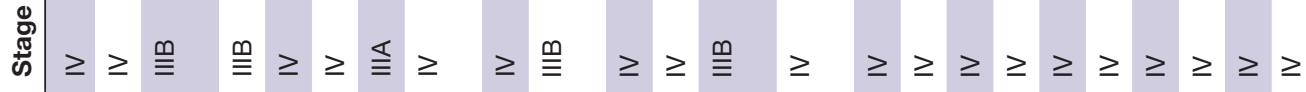
高

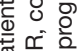




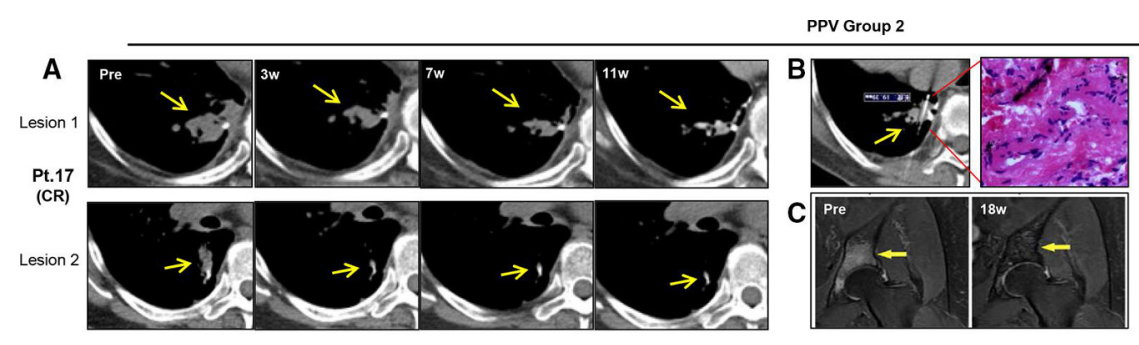

E
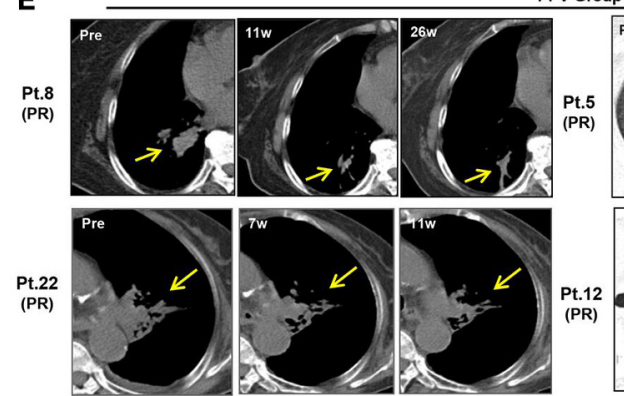
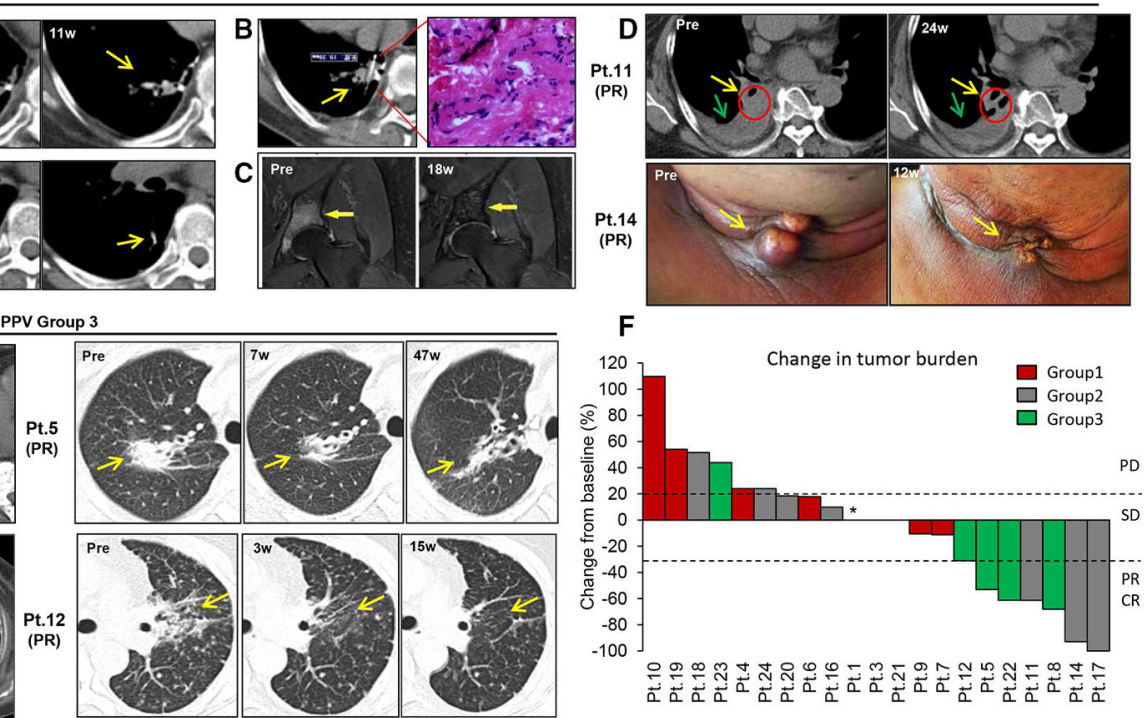

Figure 2 Patient clinical responses following personalized neoantigen peptide vaccination. (A) CT scans showing regression of two lung lesions from complete responder patient 17. (B) Tissue biopsy confirmed that the remaining lung CT signal was composed of only fibrotic tissue containing no viable tumor cells. (C) Patient 17 bone metastases evaluated by T2-weighted MRI was absent 18 weeks after the start of neoantigen vaccination (yellow arrow). This bone metastasis was considered as a non-targeted lesion according to RECIST (V.1, bone lesion measurability). (D) Two additional patients in group 2 had objective clinical responses to PPV. Patient 11 experienced lung tumor regression in addition to resolution of obstructive atelectasis 24 weeks after PPV initiation (yellow arrow and red circle), while a pneumothorax showed no change (green arrow). Subcutaneous neck metastases of patient 14 showed significant regression 12 weeks after the start of PPV treatment (yellow arrow). (E) CT scans showing lung tumor regressions in patients $5,8,12$, and 22, all of whom had partial clinical responses following PPV treatment. (F) Change in overall tumor burden of PPV study patients 3 to 4 months post-PPV compared with pretreatment baseline. Response of patients 9, 19 and 20 were assessed at 6-8 weeks after PPV initiation due to on-treatment death from disease progression. *Patient 1 developed pleural effusion at 12 weeks. CR, complete response; PD, progression disease; PPV, personalized NeoAg peptide vaccination; PR, partial response; RECIST, Response Evaluation Criteria In Solid Tumors; SD, stable disease.

\section{NeoAg-specific T cell responses against shared EGFR mutations}

To better understand the nature of the clinical responses, sequential immune monitoring was performed on peripheral blood mononuclear cells (PBMCs) collected from 20 study patients pre-PPV and post-PPV (online supplemental methods). Vaccine-induced immune responses were screened by stimulating individual patient PBMC with pools of their immunizing peptides and measuring specific interferon-gamma (IFN- $\gamma$ ) secretion by ELISA (online supplemental figure S6A,B). Using this initial assay, peptide pool-specific reactivity was detected in five patients, all of which harbored tumor-associated EGFR mutations. Peptide deconvolution revealed individual NeoAg peptide-specific IFN- $\gamma$ responses in five additional patients (online supplemental figure S6A); online supplemental table S2). Based on these results, an Immune Response ComboScore (IRC) was calculated that accounted for the breadth, intensity, and persistence of NeoAg-specific IFN- $\gamma$ responses in peripheral blood over 3 months of PPV (online supplemental methods, figure $3 \mathrm{~A}$ ). Of the 10 patients with negative immune response scores (IRC=zero), six patients were from group 1 , and only two of the patients had experienced an objective clinical response. By contrast, 8 of the 10 patients with positive IRCs were from groups 2 or 3 , which underscored the observation that patients with EGFR-mutated tumors generated notably better NeoAg-specific T cell responses than patients whose tumors expressed WT EGFR (online supplemental figure S6C). Among the 10 patients showing positive vaccine-induced immune responses were five of the seven clinical responders and five of the six patients demonstrating PFS $>9$ months (figure $3 \mathrm{~A}$ ).

IFN- $\gamma$ enzyme-linked immunospot (ELISPOT)-based assays confirmed that EGFR mutations constituted the dominant targets of NeoAg-specific $\mathrm{T}$ cell responses in the five responding patients for which vaccine-induced responses were observed. While patient 11 generated a moderate IFN- $\gamma$ response to a mutated AQP12A(L28R) NeoAg peptide restricted to HLA-A*0301, they did not generate a detectable response against an HLA-A*0201restricted EGFR(H773L) NeoAg vaccine peptide (online supplemental figure S7). By contrast, three different responding patients (patients 5, 8, and 14) generated robust immune reactivity against the $\mathrm{A} * 1101$-restricted peptide KITDFGRAK, encompassing the highly shared EGFR-L858R mutation (figure 3B). Complete responder patient 17 demonstrated a similarly strong response against the HLA-C*1502-restricted, T790M-containing peptide LTSTVQLIM. ELISPOT and HLA/peptide tetramer staining assays confirmed antigen-specific $\mathrm{CD} 8^{+}$ $\mathrm{T}$ cell responses against both of these NeoAg peptides, 

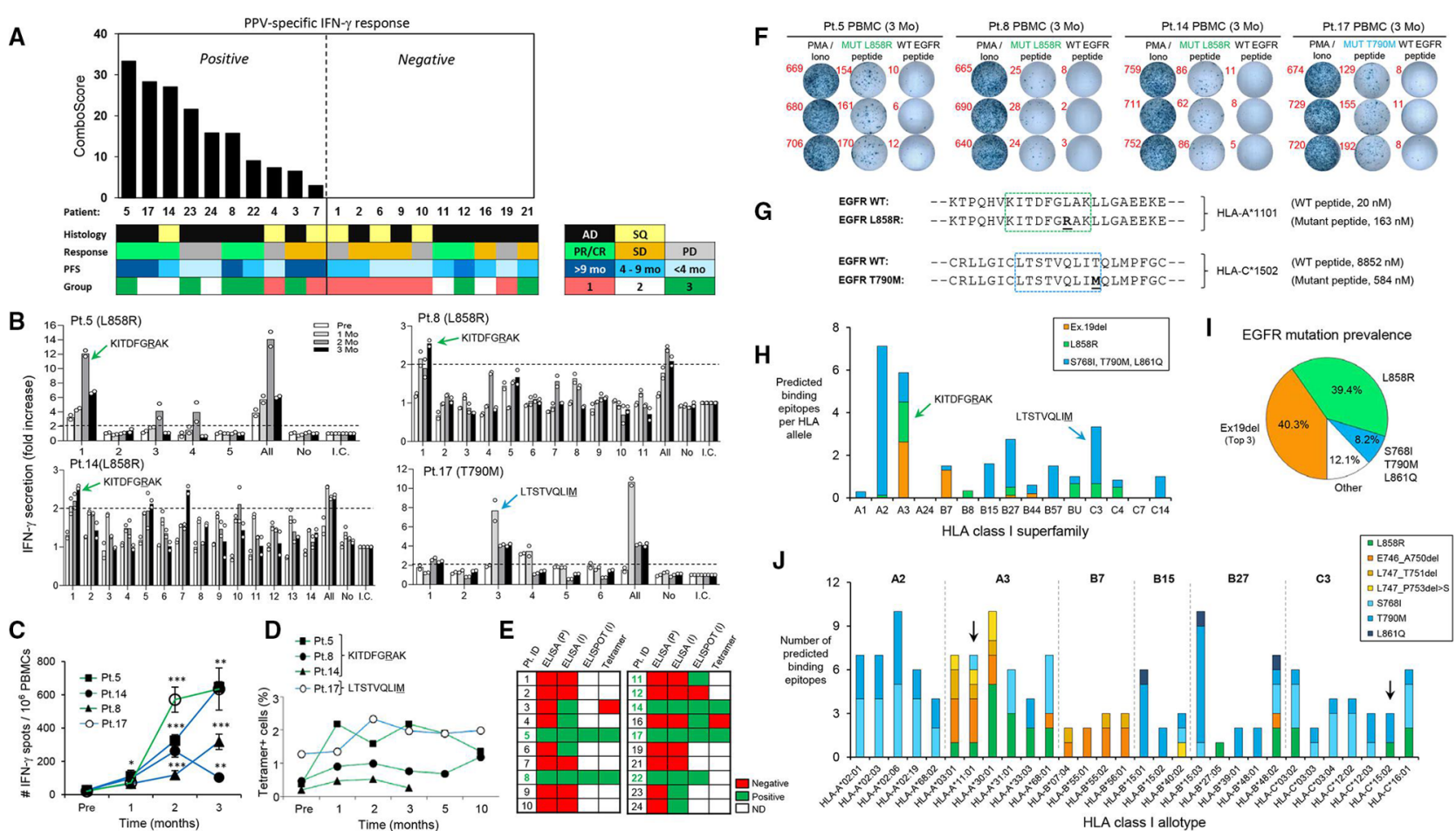

G
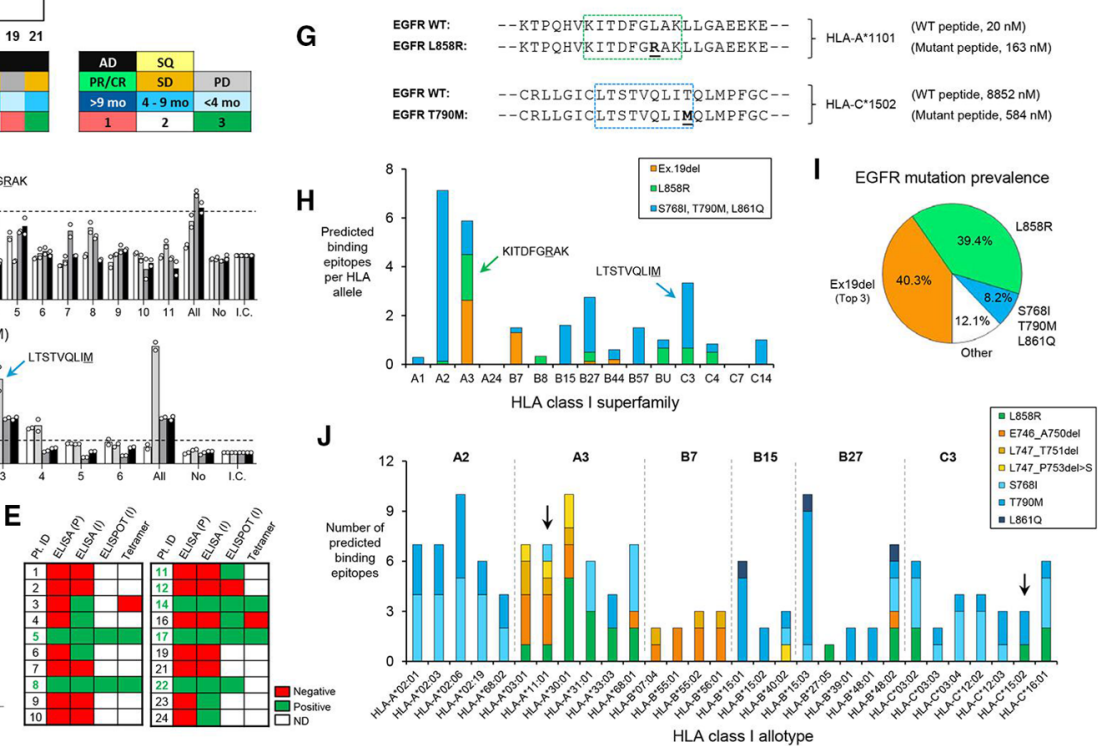

Figure 3 EGFR neoantigen peptides are immunogenic, shared and show distinctive HLA class I binding preferences. (A) Summary figure showing the total vaccine peptide-specific immune reactivity for each patient (IFN-gamma ComboScore, see Methods) along with their associated group, histology, and clinical outcome. (B) Deconvolution of individual vaccine peptide reactivities by IFN- $\gamma$ ELISA for patients 5, 8, 14 and 17 revealed dominant immune responses in patients 5, 8, and 14 against the HLA-A*1101 restricted EGFR-L858R peptide KITDFGRAK. Complete responder patient 17 similarly showed a dominant response against the HLA*C1502 restricted EGFR-T790M peptide LTSTVQLIM. Individual peptide reactivities for other PPV study patients are shown in supplemental data (online supplemental figure S6). (C and D) Summaries of IFN- $\gamma$ ELISPOT assay (three replicates) and HLA tetramer-based staining determined that PBMC frequencies of HLA-A*1101/KITDFGRAK-specific CD8 ${ }^{+} T$ cells in patients 5, 8 and 14, and HLA-C*1502/LTSTVQLIM-specific CD8 ${ }^{+} T$ cells in patient 17 increased significantly over the course of PPV. ${ }^{*} P<0.05 ;{ }^{* *} \mathrm{p}<0.01 ;{ }^{* \star *} \mathrm{P}<0.001$. (E) Summary of results of immune monitoring of study patients. Patient ID in green font indicates responding patients. (F) ELISPOT assay showed post-PPV PBMC from patients 5, 8, 14, and 17 specifically recognized mutated EGFR neoantigen peptides but not the corresponding wild-type (WT) EGFR peptides. (G) EGFR protein sequences and predicted HLA peptide binding affinities of the mutant EGFR-L858R (green) and T790M (blue) peptides and corresponding WT epitopes. ( $\mathrm{H}$ and I) Neoantigens derived from the most prevalent EGFR mutations, L858R (green) and exon 19 deletions (Ex19del, orange) show distinctive binding preferences for HLA class I allotypes within the A3 superfamily, whereas other less prevalent EGFR point mutations (S768I, T790M, and L861Q, blue) show binding preferences for HLA-A2 and C3 superfamily members. (J) Expanded view showing individual HLA class I allotypes with the highest number of predicted binding EGFR neoantigens ( $<500 \mathrm{nM}$ affinity) for the most prevalent shared EGFR mutations in lung cancer. Black arrows indicate the $A^{*} 1101$-restricted KITDFGRAK peptide and $C^{*} 1502$-restricted LTSTVQLIM peptide. Statistical comparisons were measured compared with pretreatment. Two-tailed unpaired t-tests or two-way analysis of variance test with multiple group comparison adjustment (Dunnett's test) were used to analyze the statistical significance between groups. $\mathrm{P}<0.05$ was considered significantly different. EGFR, epidermal growth factor receptor; ELISPOT, enzyme-linked immunospot; HLA, human leukocyte antigen; I, individual peptide; IFN- $\gamma$, interferon-gamma; ND, not determined; P, pooled peptides; PBMC, peripheral blood mononuclear cell; PPV, personalized NeoAg peptide vaccination.

with both assays generally demonstrating progressive incremental increases in $\mathrm{T}$ cell frequencies for up to 3 months during the immunization period (figure 3C,D; Online supplemental figure S7 and S8). Results of immune response monitoring of study patients is summarized in figure 3E. Importantly, the vaccine-induced T cells from responding patients were capable of functionally distinguishing between WT EGFR peptides and L858Rmutated or T790M-mutated NeoAg peptides (figure 3F).

Patient 22 (PR) generated a robust IFN $\gamma$ response against an A*0201-restricted FGFR1(R734W) peptide while also generating a response against a long
DRB1*0901-restricted NeoAg peptide (MASVDNPLMCRLLGICL) containing the compound EGFR mutation H773L/V774M. Similarly, patients 8 and 16 both generated immune responses against a long HLA class II restricted EGFR NeoAg peptide (HVKITDFGRAKLLGAEE) containing the L858R mutation (online supplemental figure S7). However, three other patients (patients 5,12 , and 14) vaccinated with the same peptide did not generate detectable immune responses, despite sharing HLA class II allotypes with patients 8 and 16. Interestingly, of the four L858R/HLA-A* $1101^{+}$patients immunized with the KITDFGRAK peptide, patient 16 (SD) was 
the only patient who failed to generate a robust $\mathrm{CD} 8^{+} \mathrm{T}$ cell response against this NeoAg and was also the only one of the four patients that did not experience an objective clinical response. Collectively, these results provide evidence that multiple EGFR mutations can be immunogenic targets of NeoAg vaccine-specific $\mathrm{CD} 4^{+}$and $\mathrm{CD} 8^{+} \mathrm{T}$ cell responses that are associated with clinical responses in NSCLC patients.

The KITDFGRAK NeoAg peptide target is predicted to bind HLA-A*1101 with somewhat lower affinity $(163 \mathrm{nM})$ than its corresponding WT peptide $(20 \mathrm{nM})$ but falls within the range observed for moderate-affinity HLA binders (figure 3G). By contrast, since the EGFRT790M mutation converts the peptide C-terminal anchor from a polar threonine residue to a hydrophobic methionine residue, binding of the NeoAg LTSTVQLIM peptide to HLA-C*1502 is strongly favored over the WT peptide (figure 3G). A global survey of HLA peptidebinding preferences revealed a striking skewing of EGFR NeoAg presentation at the HLA superfamily level: while L858R and Exon 19 deletion mutations, which together comprise $>80 \%$ of all EGFR mutations, produce NeoAgs with elevated basic amino acid content favoring binding primarily to A3 superfamily members including HLAA*1101, NeoAgs containing shared S768I, T790M, and L861Q mutations are more hydrophobic and are thus favored to bind members of the A2, B15, B27 and C3 superfamilies, which includes HLA-C*1502 (figure $3 \mathrm{H}-\mathrm{J}$ ) ${ }^{25}$ By contrast, HLA class I allotypes within the A1, A24, B8, and C7 superfamilies are not expected to bind and present most shared EGFR NeoAgs (online supplemental figure S9A,B). Conversely, HLA class II molecules are predicted to bind peptides containing a wide array of EGFR mutations, and class II superfamilies are not predicted to possess skewed binding preferences with the possible exception of the DP1 and DP3 allotypes (online supplemental figure S9C). ${ }^{26-28}$

\section{PPV drove proliferation and tumor infiltration of EGFR NeoAg- specific T cells}

Patient 5 received a vaccine containing a total of five NeoAg peptides (four short and one long), all of which were directed against the EGFR (L858R) mutation (online supplemental table S2). This group 3 patient received an extended course of 23 consecutive weekly immunizations and experienced a PR that was still ongoing at $>20$ months, providing a unique opportunity to analyze the long-term dynamics of their NeoAgspecific immune response. An A*1101/KITDFGRAK tetramer was used to sort EGFR(L858R)-specific CD8 ${ }^{+} \mathrm{T}$ cells from PBMC drawn 12 months following the initiation of PPV (online supplemental figure S8 and S10A). Sorted NeoAg-specific cells then underwent single-cell $\mathrm{TCR} \alpha / \beta$ sequencing, from which 52 high-confidence T-cell receptor (TCR) clones were identified $\left(\mathrm{Tet}^{+}\right.$, see online supplemental methods). Tumor biopsies taken at pretreatment or at 12 months post-PPV also underwent TCRV $\beta$-CDR3 sequencing of tumor-infiltrating lymphocytes (TILs), allowing for a detailed comparison of CDR3 frequencies in the peripheral blood and tumor compartments prior to and postimmunization. As shown in online supplemental figure S10B, CDR3 clones present in both prevaccination PBMC and TIL were present at higher frequencies within the blood, including the L858R-specific $\mathrm{Tet}^{+}$clones. By contrast, postimmunization samples showed only half the number of CDR3 clones overlapping between blood and TIL, but these clones were present at significantly higher frequencies within the TIL compartment. Importantly, NeoAgspecific $\mathrm{Tet}^{+}$clones demonstrated substantial increases in frequency in both the PBMC and TIL compartments postimmunization, including 13 new clones not detected in pretreatment samples (online supplemental figure S10B,E). Comparison of CDR3 sequences in peripheral blood pre-PPV and post-PPV showed that while a subset of T cells (including $\mathrm{Tet}^{+}$clones) increased after immunization, most other $\mathrm{T}$ cell clones showed a corresponding decrease in frequency. By contrast, at the tumor site nearly all CDR3 clones showed increased frequencies

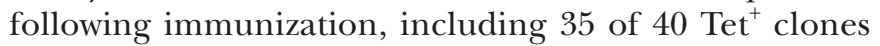
(online supplemental figure S10B).

One striking feature revealed by this analysis was that 40 of the $52 \mathrm{Tet}^{+}$clones sorted postvaccination were already present at elevated frequencies in preimmunization PBMC and TIL samples, strongly supporting the notion that spontaneous priming of EGFR NeoAg-specific cells had occurred in this patient prior to immunization. However, most of these $\mathrm{Tet}^{+}$CDR3 clones also showed significant increases in PBMC frequency over the 12 weeks that were associated with the patient's clinical response and consistent with the patient's IFN- $\gamma$ immune monitoring results (figures 2E-4E, online supplemental figure S6A,B). Interestingly, PPV also appeared to stimulate the expansion of approximately a dozen $\mathrm{Tet}^{+}$clones that were not detectable in pretreatment PBMC but showed elevated frequencies several months later in both PBMC and at the tumor site (online supplemental figure S10B,D,E). One induced $\mathrm{Tet}^{+}$clone, $\mathrm{V} \beta-\mathrm{N} 1$, increased $>500$ fold in frequency at the tumor site. TCR $\alpha / \beta$ chains from this $\mathrm{Tet}^{+}$clone were subcloned into a lentiviral vector and used to transduce PBMC-derived T cells to express TCR-N1 (online supplemental figure S10F). EGFR NeoAg-specific IFN- $\gamma$ release was confirmed by coculturing TCR-N1 transduced $\mathrm{T}$ cells with A549 tumor target cells engineered to express HLA-A*1101 and/or the KITDFG스스 minigene (online supplemental figure S10G). NeoAg-specific TCR-T cells were also shown to have the capacity to specifically lyse A*1101-positive 293 cells transduced to express full length EGFR(L858R) protein, but not WT EGFR, demonstrating that the KITDFGRAK epitope can be naturally processed and presented on HLA-A*1101 (online supplemental figure $\mathrm{S} 10 \mathrm{H}$ ). Collectively, these data provide evidence that L858R NeoAg peptide vaccination promoted a significant expansion of L858R-specific $\mathrm{CD}^{+} \mathrm{T}$ cells in patient 5 , ultimately resulting in increased frequencies of NeoAgspecific $\mathrm{T}$ cells at the tumor site. 
A

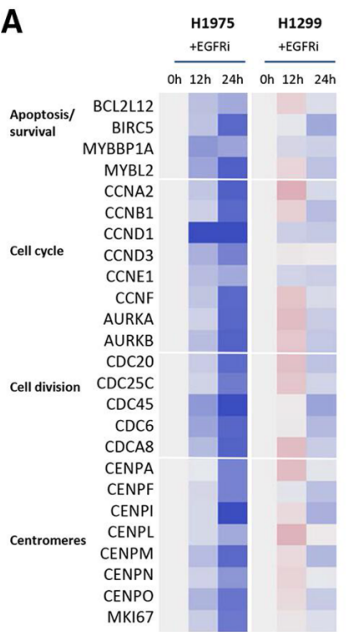

B

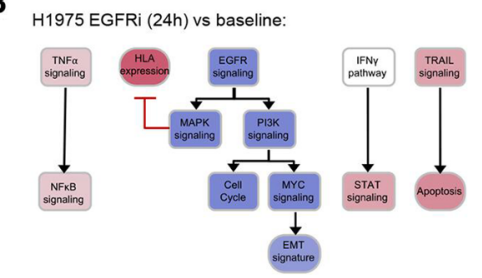

H1299 EGFRi (24h) vs. baseline:
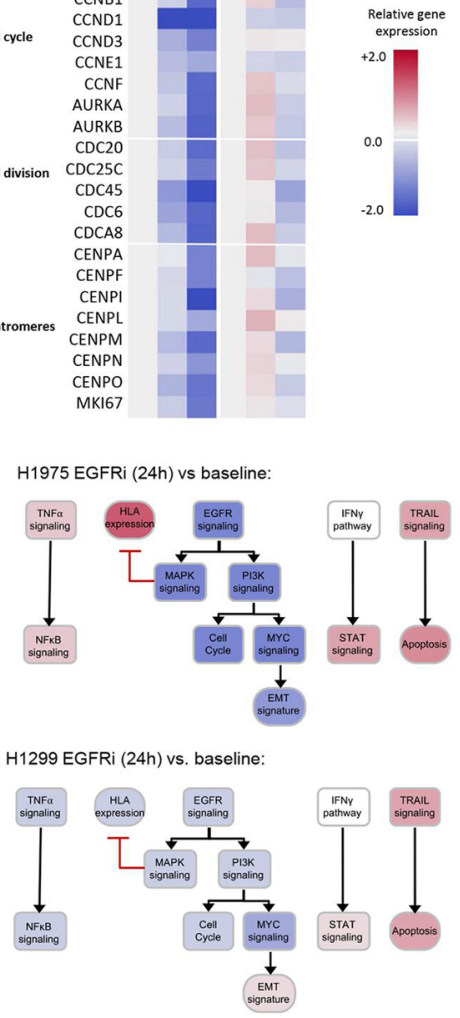

C

E

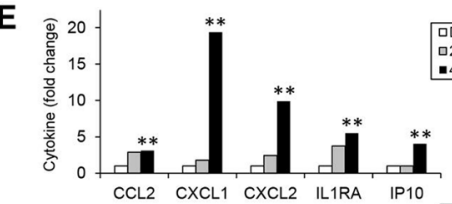

$\mathbf{F}$

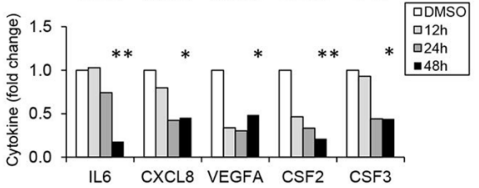

G

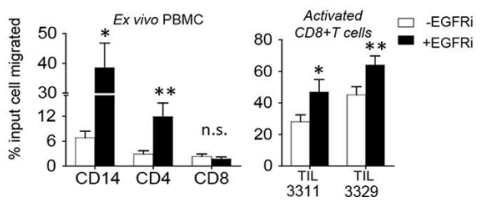

D

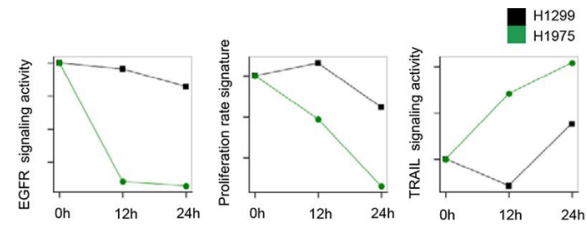

H

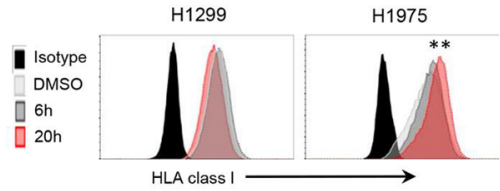

I
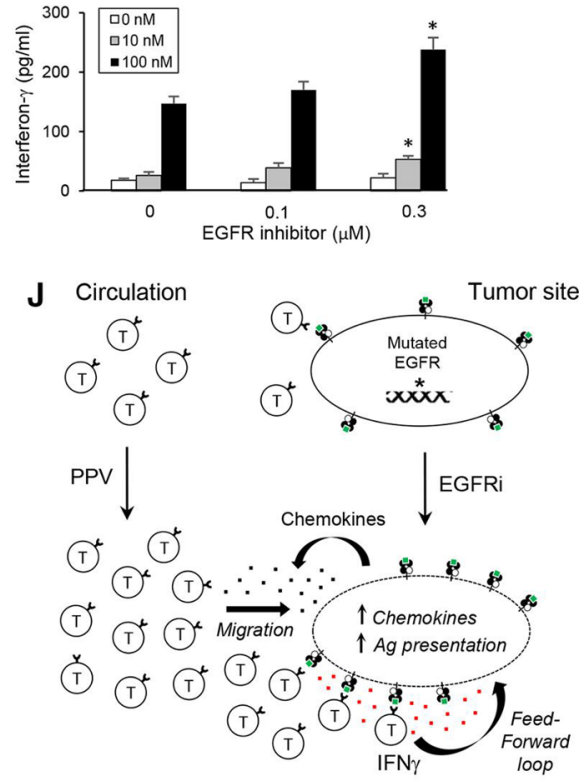

Figure 4 Immunomodulation by EGFR inhibitors (EGFRi) promotes immune cell infiltration, tumor antigen presentation, and T cell activation. H1975 (EGFR-L858R/T790M) and H1299 (EGFR-WT) cell lines were treated with the EGFRi osimertinib, and RNAseq analysis was performed at 0,12 , or 24 hours post-treatment. (A) Relative transcript expression levels of genes associated with cell division, cell cycle, apoptosis and cell survival decreased in H1975 cells following EGFRi treatment. (B) Gene expression pathway changes in EGFRi-treated H1975 and H1299 cell lines. Red: upregulation; blue: downregulation. (C) EGFRi upregulated expression of immune-related genes associated with antigen presentation and immune cell trafficking in H1975 cells. (D) EGFRi treatment of H1975 cells downregulated genes associated with EGFR signaling and proliferation rate while upregulating genes associated with TRAIL signaling. (E and F) Luminex analysis of H1975 cell supernatants confirmed changes of 10 chemokines and cytokines at the protein level. Statistical comparisons were measured compared with control. (G) Migration assay showed that EGFRi treatment of $\mathrm{H} 1975$ cells increased the migration of PBMC monocytes and CD4 ${ }^{+} \mathrm{T}$ cells and activated $\mathrm{CD}^{+}$tumor-infiltrating lymphocytes (TILs) towards H1975 cell supernatants. (H) HLA class I surface expression increased in $\mathrm{H} 1975$ but not $\mathrm{H} 1299$ cells following EGFRi treatment. (I) Tumor antigen-specific CD8 ${ }^{+} \mathrm{T}$ cells showed significantly increased IFN- $\gamma$ secretion in response to recognition of cognate antigen on EGFRi-treated H1975 cells compared with untreated cells. (J) Proposed mechanistic model to explain how EGFRi treatment might synergize with PPV to enhance immune cell trafficking and T cell activation at the tumor site. Two-tailed unpaired t-test or Mann-Whitney $U$ test was used to analyze the statistical significance between groups. $P<0.05$ was considered significantly different. ${ }^{*} P<0.05$; ${ }^{* \star} p<0.01$. EGFR, epidermal growth factor receptor; IFN- $\gamma$, interferon-gamma; PBMC, peripheral blood mononuclear cell; PPV, personalized NeoAg peptide vaccination.

\section{EGFRi can promote immune cell infiltration, antigen presentation, and $\mathrm{T}$ cell activation}

Combining PPV with concurrent EGFRi treatment induced no significant toxicities in group 3 patients. Moreover, patients showed similar clinical response rates to PPV, whether or not they continued to receive concurrent EGFRi treatment (figure 1F; online supplemental figure S2B). Group 3 patients did experience an extended overall median survival time of 13.8 months, compared with 6.0 months and 7.6 months for group 1 and group 2 patients, respectively, which was most likely attributable to the direct antitumor effects of EGFRi and the previously documented benefits of continuing EGFRi treatment beyond disease progression. ${ }^{29}{ }^{30}$ However, the observation that groups 2 and 3 patients demonstrated notably stronger $\mathrm{T}$ cell responses to NeoAg vaccination compared with group 1 patients (figure 3 and online supplemental figure S6C) led us to explore how longterm EGFRi therapy in these patients may have contributed to the promotion of antitumor immunity. 
We treated two lung cancer cell lines, H1975 (EGFR-

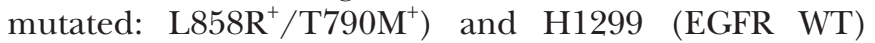
with EGFRi or DMSO, and cell supernatants and total RNA were collected at multiple time points following drug treatment. As expected, EGFRi-treated H1975 cells showed decreased EGFR signaling that was confirmed by both RNAseq and western blot analysis, in addition to decreased expression of genes associated with MYC signaling, proliferation, cell cycle, and apoptosis and survival (figure 4; online supplemental figure S11A). Examination of immune-related genes showed that EGFRi treatment increased the transcription of genes associated with TRAIL signaling and HLA class I and II antigen presentation, along with a concurrent decrease in checkpoint genes (figure 4C,D; online supplemental figure S11B,C). Transcripts encoding for several chemokines and cytokines increased or decreased following EGFRi treatment, and Luminex analysis confirmed changes to 10 of them at the protein level in cell supernatants (figure 4C,E,F). Since EGFRi treatment upregulated CXCL1, CXCL2, and CCL2, chemokines well-known to promote immune cell migration, we next examined how peripheral blood leukocytes migrated in response to EGFRi-treated or DMSO-treated H1975 cell supernatants (online supplemental figure $\mathrm{S} 11 \mathrm{~B}, \mathrm{C}$ ). Both $\mathrm{CD} 4^{+} \mathrm{T}$ cells and $\mathrm{CD} 14^{+}$monocytes from ex vivo PBMC demonstrated increased migration towards EGFRi-treated cell supernatants. Although they required prior activation to upregulate their migration capacity, $\mathrm{CD} 8^{+} \mathrm{T}$ cells also showed significantly increased migration in response to the same cell supernatants (figure 4G). Surface HLA class I surface expression was also increased in $\mathrm{H} 1975$ but not $\mathrm{H} 1299$ cells following EGFRi treatment, resulting in antigen-specific $\mathrm{CD}^{+} \mathrm{T}$ cells producing more IFN- $\gamma$ following recognition of EGFRi-treated H1975 tumor cells (figure 4H,I; online supplemental figure S11D). These results support the notion that EGFRi may promote immune cell infiltration and antigen presentation at the tumor site and contribute to the augmentation of antitumor immune responses. ${ }^{31} 32$

A model depicting how PPV and EGFRi may both contribute to antitumor immunity in distinct but complementary ways is shown in figure 4J: while PPV administration serves to stimulate the expansion of $\mathrm{NeoAg}$-specific $\mathrm{T}$ cells in the circulation, EGFRi may promote enhanced antigen presentation and chemokine secretion at the tumor site. Increased chemokines are known to augment the trafficking of immune cells including activated T cells to the tumor, where recognition of cognate tumor antigen by $\mathrm{T}$ cells can stimulate tumor cell destruction and the production of IFN- $\gamma .{ }^{33}$ Since IFN- $\gamma$ has the capacity to further upregulate antigen presentation and chemokine production $^{3435}$ (online supplemental figure S11B,C), there is the potential to initiate a 'feed-forward' loop at the tumor site to sustain the antitumor immune response (figure 4J). Further studies will be required to determine the precise contributions of EGFRi to antitumor immunity in NSCLC patients; however, the data presented here and elsewhere support the intriguing possibility that
EGFRi could contribute to the promotion of antitumor immunity either as a monotherapy or in the context of NeoAg vaccination. ${ }^{36} 37$

\section{DISCUSSION}

To our knowledge, this is the first report of a neoantigen peptide vaccine approach inducing objective clinical responses in multiple cancer patients. However, due to the overwhelming prevalence of private mutations in patients with cancer and the high level of HLA diversity in the human population, no previous vaccine study has reported immunizing multiple patients with the same shared NeoAg peptides. The high prevalence of both HLA-A*1101 and EGFR(L858R) mutations in Asian NSCLC patients predicted that $\sim 15 \%$ of our EGFRmutated patients would share this combined phenotype $^{2538}$ and provided a unique opportunity to immunize four such patients with vaccines containing EGFR NeoAg peptides in common, including the $A^{*} 1101$-restricted KITDFGRAK peptide. Remarkably, three of the four patients experienced tumor regressions within 12 weeks of NeoAg vaccination, with all three patients showing significantly increased or dominant KITDFGRAK-specific CD8 ${ }^{+}$ $\mathrm{T}$ cell reactivity during the time of the clinical responses. In demonstrating that multiple A*1101/EGFR(L858R) patients responded clinically to immunization with shared NeoAg peptides, this study provides an important first proof-of-concept in cancer vaccine studies. ${ }^{20}$

Our study also found evidence that at least two other EGFR mutations can be immunogenic in NSCLC patients, with two additional clinical responses being associated with dominant vaccine-induced $\mathrm{CD} 4^{+}$or $\mathrm{CD} 8^{+}$ $\mathrm{T}$ cell responses against the $\mathrm{H} 773 \mathrm{~L} / \mathrm{V} 774 \mathrm{M}$ or $\mathrm{T} 790 \mathrm{M}$ mutations, respectively (online supplemental figure S6A and S7; online supplemental table S3). Although T790M has a low prevalence in primary NSCLC, it develops frequently as a resistance mechanism to first-line EGFRi therapy. ${ }^{39-41}$ The T790M-containing LTSTVQLIM peptide was the dominant NeoAg target of $\mathrm{CD}^{+} \mathrm{T}$ cells in the only complete responder in our study (patient 17) and thus may constitute another promising potential shared target for the $\sim 7 \%$ of patients worldwide that express HLA-C*1502. Non-EGFR NeoAg-specific T cell responses were also detected in patients with clinical responses, most notably $\mathrm{T}$ cell responses against AQP12A(L28R) in patient 11, FGFR1(R734) in patient 22, and two distinct TP53 mutations in patients 8 and 17 (online supplemental table S2). Although our study was not designed to directly compare the immunogenicity of EGFR NeoAgs to those derived from other mutated genes, we did observe that the preponderance of immune reactivity was focused on mutated EGFR targets (online supplemental figure $\mathrm{S6C)}$. Since there is no reason to suspect that EGFRderived NeoAgs are inherently more immunogenic than those derived from other mutated tumor-associated proteins, the explanation for this finding may be related to the long-term use of EGFRi by groups 2 and 3 patients, 
as discussed further. Several non-responding patients also generated PPV-induced T cell responses, including against mutated NeoAgs derived from IDH2, MAP2K4, PIK3CA, TP53, and EGFR (746_750del). We hypothesize that the lack of clinical responses in these patients may reflect lack of NeoAg presentation by patient tumors for any number of potential reasons, including dysfunctional antigen processing, immune editing, HLA loss, or neoantigen promoter hypermethylation. ${ }^{42-44}$

Since peptide-based cancer vaccine studies have only rarely reported induction of clinical responses following immunization, it is worth discussing the unique features of our vaccination approach. To activate both the $\mathrm{CD} 4^{+}$ and $\mathrm{CD}^{+}$arms of $\mathrm{T}$ cell mediated immunity, we chose to immunize patients with mixtures of long and short NeoAg peptides, often including multiple peptides (up to six) against the same somatic mutation (online supplemental table S2). Peptides were solubilized and administered in isotonic saline to avoid any inhibitory long-term antigen depot effects; in order to compensate for the typically short half-life of saline-solubilized peptides, vaccinations were administered weekly. ${ }^{1620} 45$ Focusing the mutation calling on a panel of 508 cancer-associated, potential driver genes greatly simplified PPV design and also restricted the number of potential NeoAg targets identified per patient. Although a significant limitation of our study, this driver mutation-focused approach allowed for EGFR mutations to emerge as shared NeoAgs with promising therapeutic potential. For a vaccine adjuvant, we employed topically applied Imiquimod cream, a TLR7 agonist known to be moderately effective in activating local antigen-presenting cells. Based on the breadth, magnitude, and timing of the NeoAg-specific $\mathrm{T}$ cell responses observed, our interpretation is that the immunization approach was more effective at boosting $\mathrm{T}$ cell responses that had previously been spontaneously primed in patients, while demonstrating limited efficacy for priming new $\mathrm{T}$ cell responses. Incorporating more potent and promising vaccine adjuvants such as polyI:C, anti-CD40, or STING agonists into future peptide vaccine formulations may help to address this lack of de novo $\mathrm{T}$ cell priming. Nevertheless, PPV-mediated stimulation of pre-existing $\mathrm{T}$ cell responses were associated with multiple clinical regressions in our study, a finding that strongly supports NeoAg immunoreactivity prescreening to guide future vaccine design for NSCLC patients. ${ }^{46}$

It has been previously shown that continuation of EGFRi therapy following the development of resistance can provide a survival benefit to NSCLC patients, supported by evidence that continuation of EGFRi therapy exerts a selective pressure that inhibits more aggressive EGFRisensitive clones and allows only more indolent cells to proliferate. ${ }^{29} 30$ This would support the notion that the extended survival and clinical responses observed for group 3 patients was most likely attributable to the EGFRi therapy and not to the NeoAg vaccine. However, the seven objective clinical responses and similar response rates observed in group 2 and group 3 patients strongly suggest that NeoAg peptide vaccination was primarily responsible for reversing tumor progression in these patients. Future studies will be required to determine the precise role that EGFRi therapy may play in promoting the antitumor immunity observed in NSCLC patients, but data presented here and in other studies support this notion. ${ }^{36} 37$

One of the more notable findings in this regard was that patients previously treated with EGFRi generated stronger NeoAg vaccine-induced immune responses. One possible explanation is that first-line EGFRi monotherapy may initially induce 'immunogenic' tumor cell death leading to spontaneous cross-priming of NeoAg-specific T cells, ${ }^{478}$ which can then be boosted subsequently with NeoAg vaccination. It remains to be determined if EGFR NeoAg-specific T cell priming is favored over other tumorassociated NeoAgs; however, EGFRi drugs are known to bind irreversibly to mutated EGFR target proteins, which could conceivably impact their processing and subsequent NeoAg presentation by both APCs and tumor cells. ${ }^{49}$ It should be mentioned here that several of the responding patients did generate measurable antigen-specific $\mathrm{T}$ cell responses against private $\mathrm{NeoAg}$ vaccine peptides (online supplemental figure $S 6, S 7$ ), and we cannot discount their potential contribution to the clinical responses observed.

Although significant challenges to personalized NeoAg identification remain to be addressed, the results presented provide encouraging evidence that $\mathrm{NeoAg}$ vaccine approaches can reverse tumor progression within subsets of cancer patients harboring defined mutations and specific HLA class I allotypes. Based on the known prevalence of HLA-A*1101 and L858R mutations, the KITDFGRAK NeoAg peptide is estimated to be presented by up to $8.4 \%$ of Asian and $1.2 \%$ of North American NSCLC patients, making it one of the most widely shared NeoAgs in cancer (online supplemental table S4) ${ }^{25} 385051$ We show that multiple other EGFR mutations can also be immunogenic for patients expressing specific HLA haplotypes and that there is a natural antigen presentation 'synergy' between the most prevalent EGFR mutations and the HLA-A3 superfamily of class I allotypes, findings that have important practical implications for future vaccine development and patient selection. It is also important to note that tumor burden and pleural effusion were both independently associated with worse OS of our study patients (figure $1 \mathrm{E}$ and not shown), implying that immunization of earlier stage NSCLC patients, perhaps even prior to the development of EGFRi resistance, may be associated with better clinical outcomes. Although the results of this phase I trial are encouraging, the concepts presented here will need to be confirmed and validated in the context of larger, randomized clinical trials. However, the data gleaned from this small initial NeoAg vaccine trial provide a compelling rationale to initiate such future studies.

\section{METHODS}

\section{Data reporting}

No statistical methods were employed to predetermine patient sample numbers. The study was not randomized, 
and some investigators were not blinded during experiments and outcome assessments.

\section{Clinical trial design and treatment}

Between November 2016 and December 2018, a single-arm trial was conducted at Tianjin Beichen Hospital in China. Primary endpoints of the trial were safety and tolerability, and feasibility of the personalized approach, and secondary endpoints were antitumor immune reactivity and clinical response. Feasibility of the PPV approach was assessed by whether personalized neoantigens could be successfully identified from patients' tumor mutational analysis and multiepitope peptide vaccines designed for potential clinical application. All patients provided written informed consent before enrolling in the study.

\section{Patient eligibility}

Twenty-nine patients with stage III-IV NSCLC were enrolled in this clinical study of PPV, and 24 were successfully immunized ( 18 adenocarcinoma and 6 squamous cell carcinoma). The study patients were selected according to the following inclusion criteria: adult patients aged 18 years or more; clinical assessments classified all patients with NSCLC stage III/IV according to NCCN Clinical Practice Guidelines in Oncology, V.3.2016, Non-Small Cell Lung Cancer and the Eighth Edition Lung Cancer Stage Classification; NSCLC diagnosis was confirmed by biopsy and pathological assessment; patients experienced disease recurrence after failing conventional treatments including surgery, chemotherapy, radiotherapy and/or EGFRi therapy, and had no active treatments; patients showed good or moderate Eastern Cooperative Oncology Group performance status (PS $\leq 3$ ); patients were undergoing no other concurrent immunotherapies; pretreatment biopsy samples were available and showed at least one genetic mutation; patients had a life expectancy of $>3$ months. Patients were excluded if they: were pregnant or lactating; had known or suspected autoimmune disease, or other immune system disease; had systemic cytotoxic chemotherapy or experimental drugs for treatment of metastatic NSCLC within 4 weeks prior to first dose of personalized vaccine (not including EGFRi); had participated in any other clinical trial involving another investigational agent within 4 weeks prior to first dose of personalized vaccine; had liver or kidney dysfunction, severe heart disease, coagulation dysfunction, or hematopoietic impairment; had any active infection requiring systemic treatment; andsuffered from other current malignancies either in progress or treated within the past 5 years. Pretreatment tumor biopsies were required for the trial, and post-treatment biopsies were optional and required additional patient consent. The clinical characteristics of study patients are shown in online supplemental figure S3A, and the EGFRi treatment histories of the 16 EGFR-mutated patients are shown in online supplemental figure S3B.

\section{Generation of personalized neoantigen vaccines}

Somatic mutational analysis

Patient tumor specimens were obtained by fine-needle biopsy of tumor sites in the lung or lymph node. Tumor biopsies from individual patients underwent DNA sequencing using a 508 gene panel, in conjunction with standard clinical and pathology laboratory test procedures at Tianjin Beichen Hospital (Tianjin, China). This genotyping panel was designed to detect shared, tumorassociated driver mutations within 508 cancer-associated genes (online supplemental table S1). Tumor DNA was extracted from biopsy samples according to the instructions of the TIANamp Genomic DNA Kit (Tiangen, China) and detected by Hiseq X-10 (Illumina, USA), which profiled using exon capture by hybridization followed by next-generation DNA sequencing (Hengjia Medical Laboratory, Tianjin, China). For somatic mutation calling, analyses of next-generation sequencing data of tumor and matched PBMCs (as source of normal germline DNA) from the patients were used to identify the specific coding sequence mutations, including singlenucleotide, dinucleotide or trinucleotide variants that lead to single amino acid missense mutations and small insertions/deletions (indels). Output from Illumina software was processed by the Broad Picard Pipeline to yield BAM files, which contained aligned reads (bwa V.0.7.8, aligned to the NCBI Human Reference Genome Build hg19) with well-calibrated quality scores. Somatic single nucleotide variations, somatic small insertions and deletions were all detected using Varscan2 (V.2.4.3). All indels were manually reviewed using the Integrative Genomics Viewer (V.2.4.1). All somatic mutations, insertions and deletions were annotated using Annovar (V.2013-07-28 11:32:41). Neoantigen peptides were chosen based on non-synonymous somatic mutations detected at a mutated variant allele frequency of 0.04 or higher.

\section{HLA typing}

Peripheral blood was drawn for high resolution HLA typing at the time of enrollment. HLA loci were typed via PCR sequence-based typing method employing a DNA amplification step (CapitalBio, China). Briefly, DNA was extracted from peripheral blood of patients according to the instructions of the Magic Beads DNA Extraction Kit (TANBead, China). Exons 2 and 3 of HLA class I genes (HLA-A, B, and C) and exon 2 of HLA class II $\alpha$ and $\beta$ genes (HLA-DQ and DR) were amplified and purified, and PCR products were sequenced on ABI 3730XL DNA Analyzer (Applied Biosystems, USA). Sequence chromatograms were analyzed using ATF1.5 software (Conexio Genomics, Australia).

\section{Vaccine peptide selection}

Due to the high number of somatic mutations typically found in lung cancers and the fact that the majority constitute private 'passenger' mutations, we chose to target somatic mutations detected from a focused panel of 508 tumor-associated genes. The rationale for this approach 
was twofold: (1) it would enable targeting of mutations more likely to be essential for the tumor phenotype and thus less likely to be lost through immune editing, and (2) it would increase the chances of identifying shared neoantigen targets that could potentially be beneficial to multiple NSCLC patients. Non-synonymous coding mutations detected by the 508-gene panel were translated in silico, and the resulting neoantigen sequences were assessed for predicted binding affinity to patient HLA class I and class II molecules according to the HLA-peptide prediction algorithms NetMHC4.0, NetMHCpan3.0, NetMHCpan4.0, NetMHCII2.2 and NetMHCII2.3. ${ }^{28} 52$ Neoantigen vaccine peptides were chosen primarily based on highest predicted binding affinity to the patient's HLA class I and class II molecules. However, vaccines were also designed to maximize the number of different HLA molecules engaged and minimize intra-HLA peptide competition when possible. Certain biochemical properties (such as elevated hydrophobicity or the presence of multiple cysteines), which can negatively impact the synthesizability or solubility of the immunizing peptides, were also considered. In addition, we aimed to design individual patient vaccines to contain an approximately 2:1 ratio of short to long vaccine peptides, or as close as the somatic mutation profiling and HLA/peptide binding predictions would allow. For each patient, up to 14 peptides of 9-20 amino acids in length arising from up to 12 independent mutations were selected and prioritized. A mean of 9.4 neoantigen peptides per patient were chosen for peptide synthesis, which included on average 6.5 short, HLA class I restricted peptides and 2.9 long, HLA class II restricted peptides (online supplemental table S2).

\section{Patient immunizations}

Of 29 patients enrolled into the study, three patients passed away while waiting for the vaccine peptides to be produced and were therefore unable to participate. Two additional patients were not treated since their PS increased to 4-5 during production of their vaccine peptides, which then caused them to fail to meet the inclusion criteria of $\mathrm{PS}<3$, and they went on to receive other treatment. Immunizing peptides were synthesized using standard solid-phase synthetic peptide chemistry, purified to $>98 \%$ using reverse phase high performance liquid chromatography and tested for sterility and the presence of endotoxin to ensure safety and tolerability using methodologies consistent with Good Manufacturing Practice (HengJia Neoantigen Biotechnology (Tianjin)). As shown in online supplemental table S2, 5-14 peptides per patient were synthesized, solubilized individually in sterile phosphate-buffered saline (PBS), and mixed into two separate peptide cocktails, each with two to seven short peptides and one to three long peptides in $1 \mathrm{~mL}$ total volume. Peptides binding to the same HLA allotypes were separated into different cocktails to reduce potential antigen competition. Patients received $200 \mu \mathrm{g}$ of each peptide per immunization, injected subcutaneously into the left and right extremities, and administered weekly for 12 weeks. In order to provide concurrent TLR-7 stimulation of pAPCs, Aldara cream with $5 \%$ imiquimod was applied topically as a vaccine adjuvant over the vaccine site immediately after peptide cocktail administration. Patients were permitted to continue immunizations after 12 weeks if desired and in the patient's best interest. Eleven of the 24 immunized patients continued to receive vaccinations beyond 12 weeks, as shown in online supplemental figure S1 and online supplemental table S1.

\section{Sample collection}

Serial PBMC samples were collected at pretreatment, as well as at 4, 8, and 12 weeks postvaccination. A maximum of $15 \mathrm{~mL}$ of blood was drawn per month, according to Tianjin Beichen Hospital regulations for patients with advanced-stage cancer. Collection of additional blood samples beyond the 12 weeks of the trial period were optional and required additional patient consent. Extra blood samples were collected from patients 5, 8, and 17 , who had all experienced clinical objective responses. Viable PBMCs were collected and stored at $-80^{\circ} \mathrm{C}$. Collection of postvaccine tumor biopsies was optional but not required in this trial due to the invasive nature of the procedure, uncertain feasibility, and the general reluctance of most patients. Tumor tissues obtained for further bulk $\mathrm{T}$ cell receptor V $\beta$ CDR3 sequencing analysis were collected from PPV trial patient 5 after providing written informed consent.

\section{Tumor response evaluation criteria}

Objective tumor response assessments were made according to the RECIST (V.1.1) guidelines. We used CT and/or MRI scans to measure selected target lesions (online supplemental table S5). Patients were required to perform at least one pretreatment scan for baseline measurements and another scan at 3-4 months postvaccine for response assessment. Additional patient scans were taken monthly during the first 12 weeks of vaccination if feasible. Target lesions with a minimum size of $10 \mathrm{~mm}$ ( $15 \mathrm{~mm}$ for malignant lymph nodes) were measured in the longest diameter by three different radiologists, with the mean of the three independent measurements used for clinical assessments. A maximum of two target lesions per organ were measured, with the two largest lesions selected, up to a maximum of five lesions in total. Tumor burden was calculated as the sum of the diameters of all target lesions (online supplemental figure S4B). Clinical responses were evaluated as follows: CR, complete disappearance of all target lesions; PR, partial response, defined as a $30 \%$ decrease in the sum of diameters of target lesions; PD, progressive disease, defined as a minimum $20 \%$ increase in the sum of diameters of target lesions or the appearance of new lesions; and SD, stable disease, defined as a change in tumor burden insufficient to qualify for PR or PD. Clinical responses were assessed 3-4 months following the date of the first immunization. 


\section{Clinical trial statistical plan}

Statistical analysis was primarily descriptive, including enumeration of patients who experienced any adverse events. All statistical tests were two sided with an alpha level of 0.05 . CIs to be evaluated were constructed with a significance level of 0.05 . Additional exploratory analyses of the data were conducted as deemed appropriate.

\section{Analysis of primary endpoints}

Treatment-associated adverse events were analyzed based on those categorized and graded according to the National Cancer Institute Common Terminology Criteria for Adverse Events (V.4.0). During the first 12 weeks of vaccine treatment and continued vaccination beyond 12 weeks, safety assessments were performed starting on the day of each vaccination until the next injection. Safety assessments were performed every 2 months for patients with extended follow-up time. Disease-associated symptoms that were present at baseline (pretreatment) were not reported unless they worsened after vaccination.

\section{Analysis of secondary endpoints}

Measurements of the immune responses via ELISA, ELISPOT and tetramer analysis prior to the first vaccination and every 4 weeks after each vaccination were summarized descriptively. Determinations of PFS and OS for enrolled subjects were calculated from the date of enrollment to disease progression and/or death or 31 December 2018, respectively. PFS and OS were summarized using the Kaplan-Meier method. All data analysis was performed during and after January 2019.

\section{Subgroup analyses}

Based on their distinct immune and clinical response profiles, we analyzed the enrolled patients according to subgroup based on WT or mutated (Mut) EGFR mutation status, and if EGFR inhibitor use was continued or stopped prior to the start of immunization. These groups were defined as EGFR WT-PPV only (group 1), EGFR Mut-PPV only (group 2) and EGFR Mut-PPV+EGFRi (group 3).

\section{ELISA and ELISPOT assays}

PBMCs collected prior to the start of immunization and at different time points following PPV were isolated by Ficoll density gradient centrifugation and counted in the presence of trypan blue dye to evaluate viability prior to cryopreservation. For ELISA analysis, $5 \times 10^{5}$ PBMCs in RPMI-1640 containing $10 \%$ fetal bovine serum (FBS) were added to each well of 96-well plate with a total volume of $250 \mu \mathrm{L}$. PBMCs were cultured in the presence of vaccine peptide pools, individual vaccine peptides, or irrelevant control peptides $(7.5 \mu \mathrm{g} / \mathrm{mL})$ along with $300 \mathrm{IU} / \mathrm{mL}$ interleukin-2 (IL-2) in a $37^{\circ} \mathrm{C}$ humidified incubator with $5 \% \mathrm{CO}_{2}$ for 5 days. Following 24 hours of peptide restimulation, the IFN- $\gamma$ concentration of cell supernatants was measured using a human IFN- $\gamma$ ELISA kit (Dakewe, China), according to the manufacturer's instructions. A level of IFN- $\gamma$ secretion twofold or greater over background signal with irrelevant control peptide was considered to be a positive immune response for initial screening.IRC that considered breadth, intensity, and persistence of vaccine-induced immune responses were calculated for each patient as follows: ComboScore=sum of fold increases $>2$ for all vaccine peptides at all postvaccine time points $(4,8$, and 12 weeks). For ELISPOT assays, $2.5 \times 10^{5}$ PBMCs were prepared in RPMI-1640 containing $10 \%$ FBS with a total volume of $150 \mu \mathrm{L}$ for each well in a 96-well plate. Ex vivo PBMCs were stimulated in triplicate with individual vaccine peptides at a final concentration of $10 \mu \mathrm{g} / \mathrm{mL}$, and plates were incubated at $37^{\circ} \mathrm{C}$ in humidified incubator with $5 \% \mathrm{CO}_{2}$ for 36 hours. For patients 11, 12, 16, and 22, in vitro PBMC stimulation was performed for 12 days in the presence of individual ( $2 \mu \mathrm{g} /$ $\mathrm{mL})$ or pooled vaccine peptides $(2 \mu \mathrm{g} / \mathrm{mL}$ each $)$ and IL-2 (50 IU/mL, Sihuan Biopharmaceutical, Beijing, China), IL-4 (5 ng/mL, Cat. No.: 101-04, PrimeGene), IL-7 (5 ng/ $\mathrm{mL}$, Cat. No.:101-07, PrimeGene), and IL-15 (5 ng/mL, Cat. No.:101-15, PrimeGene) prior to performing IFN- $\gamma$ ELISPOT. Spot detection was performed using a Human IFN- $\gamma$ ELISpot PRO kit (MABTECH, USA) and normalized to the number of IFN- $\gamma$ spots detected per $10^{6} \mathrm{PBMC}$.

\section{Tetramer staining and flow cytometric analyses}

Selected custom phycoerethrin (PE) or Allophycocyanin (APC)-conjugated HLA-peptide tetramers (Baylor College of Medicine, USA; MBL, Japan) were successfully generated (online supplemental figure S9A). PBMCs were thawed and resuspended in RPMI-1640 containing $0.5 \%$ FBS. One hundred microliters of PBS- $1 \%$ BSA containing fluorophore-conjugated HLA/peptide tetramer (1:50 dilution) was added to $5 \times 10^{5}$ PBMCs and incubated at room temperature for $20 \mathrm{~min}$ in the dark. Cells were washed with PBS-1\% BSA, stained with Fluorescein isothiocyanate (FITC)-conjugated or Phycoerythrin (PE)-conjugated anti-CD8 mAbs (Biolegend, USA, 1:200 dilution), and incubated for $15 \mathrm{~min}$. Cells were then washed and resuspended in $400 \mu \mathrm{L}$ PBS- $1 \%$ BSA for flow cytometric analysis (LSRFortessa X-20 Analyzer).

\section{Bulk $T$ cell receptor V $\beta$ CDR3 sequencing analysis}

DNA samples were extracted from patient PBMC or pretreatment and post-treatment tumor biopsies from patient 5 using a DNA extraction kit (Qiagen, USA), followed by library construction with two rounds of PCRbased amplification. CDR3 fragments were first amplified using specific primers for each $\mathrm{V}$ and $\mathrm{J}$ gene, and target fragments of multiplex-PCR products were purified using magnetic beads (A63882, Beckman, Germany). Next, PCR was performed using universal primers, and target fragments 200-350 bp were retrieved and purified by QIAquick Gel Purification Kit (Qiagen, USA). PCR products were then sequenced using the Illumina X10 platform. Single-read CDR3 sequences were eliminated, and the remaining sequences were analyzed to evaluate TCRV $\beta$ IMGT clonality of patients before and after treatment, as previously described. ${ }^{53}$ 


\section{Single-cell T cell receptor sequencing}

HLA-A*1101/KITDFGRAK Tetramer ${ }^{+} \mathrm{CD}^{+}{ }^{+} \mathrm{T}$ cells from post-PPV PBMCs of patient 5 were sorted using a flowbased cell sorter (BD FACSAria III, USA) and imaged by confocal microscope (Leica SP8, Germany). Sorted Tet $^{+}$cells were adjusted to $1 \times 10^{6}$ cells per $\mathrm{mL}$ in PBS and loaded on a Chromium Single Cell Controller (10X Genomics, USA) to generate single-cell gel beads in emulsion (GEMs) using a Single Cell 5' Library and Gel Bead Kit (10X Genomics, USA). Captured cells were lysed, and released RNA was barcoded through reverse transcription to produce barcode-containing cDNA in individual GEMs as per the manufacturer's introductions. $\mathrm{V}(\mathrm{D}) \mathrm{J}$ sequences were enriched by nested PCR amplification with specific primers targeting conserved TCR sequences. Sequencing was performed on an Illumina NovaSeq platform with $150 \mathrm{bp}$ (PE150) paired ends. Cellranger VDJ was used for analyzing $\mathrm{V}(\mathrm{D}) \mathrm{J}$ recombination, T cell diversity, and pairing of appropriate TCR $\alpha$ and $\beta$ chain sequences for each individual $\mathrm{T}$ cell.

Single-cell sequencing of Tet+ cells resulted in the identification of 639 distinct TCR $\alpha / \beta$ pairs. However, since tetramer-based cell sorting can result in contamination with non-antigen specific T cells, we chose to select TCR clones for which a minimum of $10 \mathrm{~V} \beta-\mathrm{CDR} 3$ reads were detected, resulting in 51 unique TCR clones. Four clones with V $\beta$-CDR3 sequences that showed little or no presence in any PBMC or TIL samples were eliminated from the analysis, and five additional TCR clones with either seven or eight CDR3 reads were included based on their increasing frequencies in PBMC and/or TIL during PPV, as would be expected for a neoantigen-specific $\mathrm{T}$ cell clone.

\section{TCR cloning and validation}

The variable region sequences of TCR V $\alpha-\mathrm{N} 1$ and VB-N1 chains obtained by single cell sequencing were fused to an engineered human constant region to enhance $\alpha$ and $\beta$ chain pairing. ${ }^{54}$ These modified $V \alpha$ and $V \beta$ chain sequences were synthesized and inserted into a EFla promoter based lentiviral expression vector pCDH to create lentivirus lenti-EF1a-TCR-N1. Healthy donor PBMCs were prepared using lymphocyte separation medium (Stem Cell, Canada). T cells were isolated using the Dynabeads Human T-Expander CD3/CD28 Kit (Thermofisher, USA), mixed with $3 \mathrm{~mL}$ X-Vivo15 serum-free medium (Lonza, Switzerland) and cultured at $37^{\circ} \mathrm{C}$ in $5 \% \mathrm{CO}_{2}$ for 48 hours. Cell density was adjusted to $1 \times 10^{6} / \mathrm{mL}$ and cocultured with packaged lentivirus lenti-EF1a-TCR-N1 for 4 days at $37^{\circ} \mathrm{C}$ in $5 \% \mathrm{CO}_{2}$. TCR-N1 expression and antigen specificity was confirmed by staining with the HLA-A*1101/KITDFGRAK tetramer. A minigene encoding the KITDFGRAK peptide linked to the HLA-A*1101 cDNA through an IRES sequence was synthesized and inserted into lentiviral vector pCDH with EFla promoter to create lentivirus lentiEF1a-KIT-A11. Control lentiviral constructs included vectors that expressed either the KITDFGRAK minigene or HLA-A*1101 individually. Lentiviral-transduced A549 lung cancer cells stably expressing the gene(s) of interest were selected through purinomycin-based selection. HLA-A*1101 surface expression was confirmed by staining with an $A^{*} 1101$-specific mAb followed by flow cytometric analysis. Engineered TCR-N1-T cells were cocultured with parental A549, A549-A11, A549-KIT, or A549-A11.KIT cells $\left(20,000\right.$ target cells per well) at $37^{\circ} \mathrm{C}$ in 5\% $\mathrm{CO}_{2}$ with effector-to-target ratios of 1:1, 2.5:1, 5:1 and 10:1. Supernatants were collected after 24 hours of coculture to assess levels of IFN- $\gamma$ secretion by ELISA. Cytolytic activity of KITDFGRAK-specific TCR transduced $\mathrm{T}$ cells also was assessed using a standard chromium-51 $\left({ }^{51} \mathrm{Cr}\right)$ release assay. Target cells were labeled with $100 \mu \mathrm{L}$ of ${ }^{51} \mathrm{Cr}$ for 1 hour, then washed and plated at 2000 target cells per well in triplicate. TCR-T cells were incubated for 4 hours at various effector-to-target cell ratios with A*1101-expressing 293 embryonic kidney target cells either transduced to express full-length WT or L858Rmutated EGFR cDNA, or pulsed with WT EGFR peptide KITDFGLAK or L858R mutated KITDFGRAK peptide $(5 \mathrm{uM})$. After the incubation, supernatant was collected from the wells, and ${ }^{51} \mathrm{Cr}$ was measured with a gamma radiation counter. The percentage of specific target cell lysis was calculated, correcting for background ${ }^{51} \mathrm{Cr}$ release and relative to a maximum release as indicated by Triton $\mathrm{X}-100$ lysed target cells.

\section{Immunoprecipitation and western blot}

H1975 (EGFR-L858R/T790M) and H1299 (EGFR-WT) lung cancer cell lines (ATCC, USA) were treated with different concentrations $(0.1,1,2$, and $5 \mu \mathrm{M})$ of EGFRi Osimertinib (LC Laboratories, USA) for different time periods to optimize EGFRi treatment conditions. Cells were washed with cold PBS and lysed using lysis buffer (1\% Triton X-100, Sigma, USA) and Halt Protease Inhibitor Cocktail 100X and $0.5 \mu \mathrm{M}$ EDTA 100X (Thermo Scientific, USA). Lysates were collected and centrifuged at high speed for $30 \mathrm{~min}$ at $4^{\circ} \mathrm{C}$ prior to measuring protein concentration with a Bradford assay kit (BioRad, USA). Preclearing was performed using $10 \mu \mathrm{L}$ Pierce Protein A/G Ultra Link resin (Thermo Scientific) per sample and incubating for 2 hours at $4^{\circ} \mathrm{C}$. Immunoprecipitations were performed using the same amount of total protein and by incubating cell lysates for 18 hours at $4^{\circ} \mathrm{C}$ with the following antibodies at a 500:1 dilution: antiphosphoEGFR (EMD Millipore), anti-EGFR, Anti-p44/42 MAPK ERK1/2, anti-phospho-p44/42 MAPK ERK1/2, and anti-GAPDH (Cell Signaling Technology). Protein A/G crosslinking beads were added and incubated for 4 hours prior to washing with cold PBS. Samples were run using an SDS-PAGE gradient (8\%-16\%) gel (Invitrogen, USA). Proteins were transferred to a PVDF membrane (Thermo Scientific, USA), and blots were blocked with 5\% milk prior to incubation with specific antibodies overnight at a concentration of 1:1000. Blots were washed and incubated with peroxidase-conjugated antirabbit secondary antibody $(1: 10,000)$ (Jackson Immuno Research, USA). 
Blots were developed using the Super Signal West Pico PLUS Chemiluminescent enhanced horseradish peroxidase substrate (Thermo Scientific, USA) and visualized with X-ray film.

\section{RNA sequencing of lung tumor cell lines}

H1975 and H1299 lung cancer cells were treated with $1 \mu \mathrm{M}$ of EGFRi Osimertinib (LC Laboratories, USA) for 0 hour, 12 hours, 24 hours, or 24 hours with $500 \mathrm{U} /$ $\mathrm{mL}$ recombinant human IFN- $\gamma$ (R\&D Systems, USA) added for the last 12 hours of culture. Cells were lysed, and total RNA was prepared using an RNeasy Mini Kit (Qiagen, USA) according to the manufacturer's protocol. RNAseq was performed by the Avera Institute for Human Genetics (South Dakota, USA) as follows: total RNA was assessed for degradation on an RNA 6000 Nano chip ran on a 2100 Bioanalyzer (Agilent, USA) where the average RNA integrity score for the sample set was 9.7. Sequencing libraries were prepared using the TruSeq Stranded Total RNA Library Prep Kit (Illumina, USA) following the low sample procedure. Briefly, ribosomal RNA was depleted from total RNA, and the remaining RNA was purified, fragmented appropriately, and primed for cDNA synthesis. Bluntended cDNA was generated after first and second strand synthesis. Adenylation of the 3 ' blunt-ends was followed by adapter ligation prior to the enrichment of the cDNA fragments. Final library quality control was carried out by evaluating the fragment size on a DNA1000 chip ran on a 2100 BioAnalyzer (Agilent, USA). The average library yielded an insert size of 326 base pairs (bp). The concentration of each library was determined by quantitative PCR by the KAPA Library Quantification Kit for Next Generation Sequencing (KAPA Biosystems, USA) prior to sequencing. Libraries were normalized to $2 \mathrm{nM}$ in $10 \mathrm{mM}$ Tris-Cl, pH8.5 with $0.1 \%$ Tween 20 then pooled evenly. The pooled libraries were denatured with $0.05 \mathrm{M} \mathrm{NaOH}$ and diluted to $20 \mathrm{pM}$. Cluster generation of the denatured libraries was performed according to the manufacturer's specifications (Illumina, USA) using the HiSeq PE Cluster Kit V.2 chemistry and flow cells. Libraries were clustered appropriately with a $1 \%$ PhiX spike-in. Sequencing-by-synthesis was performed on a HiSeq2500 using V.2 chemistry with paired-end $101 \mathrm{bp}$ reads resulting in an average of 52.4 million paired-end reads per sample. Sequence read data were processed and converted to FASTQ format for downstream analysis by Illumina BaseSpace analysis software, FASTQ Generation V.1.0.0.

\section{RNA sequencing data analysis}

Quality control of cell line RNAseq data was performed using FastQC V.0.11.5, FastQ Screen V.0.11.1, RSeQC V.3.0.0, MultiQC V.1.6 and proprietary algorithms of the BostonGene platform. ${ }^{55}$ RNAseq reads were pseudoaligned using Kalisto V.0.42.4 to GENCODE V.23 transcripts. ${ }^{56}$ Transcripts with transcript type in (protein_coding, IG_C_gene, IG_D_gene, IG_J_gene, IG_V_gene, TR_C_gene, TR_V_gene, TR_D_gene, TR_J_gene) were selected, then non-coding RNA transcripts and histones and mitochondrial transcripts were removed resulting in 20,062 protein coding genes. Gene expressions were quantified as transcripts per million and $\log 2$ transformed. ${ }^{57}$ Gene expression changes in cell lines treated with EGFRi were shown as relative (log) expression normalized to untreated control cells. PROGENy V.1.4.1 was used to calculate seven pathways activity scores (EGFR, MAPK, PI3K, TRAIL, TNFa, NFkB, and JAK-STAT) ${ }^{58}$ Other pathway signature scores were calculated using ssGSEA using in-house python implementation. The pathways activity were represented as gene signatures, downloaded from mSIGdB V.6.2, ${ }^{59}$ unless other specified: 'Cell cycle signature' - HALLMARK_G2M_CHECKPOINT, 'Apoptosis signature' - HALLMARK_APOPTOSIS, MYC HALLMARK_MYC_TARGETS_V2, 'EMT signature' -HALLMARK_EPITHELIAL_MESENCHYMAL_TRANSITION, 'IFN- $\gamma$ signature' - HALLMARK_INTERFERON_GAMMA_RESPONSE, 'HLA expression' - gene set (HLA-A, HLA-B, HLA-C). Pathway score differences relative to the control were normalized to the maximum values in each pathway separately and displayed on the line plots. For schematic representation, the maximum absolute deviation of the pathway activity score change from the control point ( 0 hour) to the 24 hours time point was calculated within each pathway. Pathway colors on the schema correspond to the percent of the maximum absolute deviation. Heatmaps, dot plots, line plots, and bar plots were created using pandas V.0.23.4, matplotlib V.2.1.1 and seaborn V.0.9.0 python packages. ${ }^{60}$

\section{Luminex assay}

Duplicate samples of supernatants from untreated and $1 \mu \mathrm{M}$ EGFRi-treated H1975 and H1299 cell lines were analyzed for the presence of CCL2, CXCL1, CXCL2, CXCL8, IP10, IL1RA, IL6, VEGFA, CSF2, and CSF3 proteins using a custom Luminex kit according to the manufacturer's instructions (R\&D Systems, USA). Fifty microliters of test supernatant and the appropriate microparticle cocktails were added (1:10 dilution) to each well and incubated for 2 hours in a microplate shaker at room temperature. Plate was washed three times, and $50 \mu \mathrm{L}$ of Biotin-Antibody cocktail (1:10 dilution) was added and incubated for 1 hour, followed by three washes. After incubating with $50 \mu \mathrm{L}$ of Streptavidin (1:25 dilution) for $30 \mathrm{~min}$, the plate was washed three times, and microparticles were resuspended in buffer and the plate was read using a Luminex plate reader. EGFRi-induced changes were expressed as fold increase or fold decrease compared with measured baseline (0hour) concentrations. In cases where concentrations measured fell below the level of detection, the minimum threshold of detection according to the manufacturer was used. 


\section{T cell functional assays}

$T$ cell migration

EGFRi Osimertinib (LC laboratories, USA) at $1 \mu \mathrm{M}$ concentration was used to treat H1975 cells for 24 hours. DMSO only with media was used as a control. Cells were then washed and incubated in ImmunoCulut-XF $\mathrm{T}$ cell expansion medium (Stem Cell Technology, Canada) for 24 hours, after which cell supernatants were collected and filtered using a Millex-GS filter. Healthy donor PBMC or expanded melanoma CD8 ${ }^{+}$TILs (TIL 3311 and TIL3329) were thawed $\sim 16$ hours prior to performing the migration assay. Six hundred and fifty microliters of H1975 cell supernatant was placed at the bottom of a transwell plate (Corning, USA) and incubated with $3 \times 10^{5} \mathrm{PBMC}$ or TIL in the top well for 6 hours. Migrated cells at the well bottom were collected and stained for CD4, $\mathrm{CD} 8$, or CD14 (Biolegend) for $30 \mathrm{mins}$ at $4^{\circ} \mathrm{C}$, washed with PBS and fixed with $4 \%$ PFA. Fifty microliters of counting beads were added to each sample to obtain accurate cell counts. Samples were run on a Canto II flow cytometer and analyzed using FlowJo V.10.

\section{HLA class I quantitation and T cell antigen recognition}

H1299 and H1975 cells were treated with $1 \mu \mathrm{M}$ EGFRi Ostermitinib (LC laboratories) or DMSO control for 6 hours or 20 hours. Cells were collected and stained for total class I (W6/32-APC, Thermo-Fisher, USA), washed, and fixed in $4 \%$ PFA. Samples were run on a Canto II flow cytometer and analyzed using FlowJo V.10. H1975 cells were seeded at 50,000 cells per well in 96-well plates, and EGFRi was used to treat cells at concentrations of $0,0.1$, and $0.3 \mu \mathrm{M}$ per well for 24 hours. H1975 cells were then pulsed with 0,10 , or $100 \mathrm{nM}$ of cognate HLA-A*0101-restricted VGLL1 peptide LSELETPGKY for 1 hour prior to washing. VGLL1 peptide antigen-specific $\mathrm{CD}^{+} \mathrm{T}$ cells were then added at a 1:1 effector-to-target cell ratio and cocultured overnight. IFN- $\gamma$ in 24-hour cell supernatants was analyzed using a human IFN- $\gamma$ ELISA kit (Invitrogen, USA) and plates were read using SpectraMax M5/M5e Multimode Plate Reader.

\section{Statistical analysis}

All statistical analyses were performed using the GraphPad Prism 5 or 8 (GraphPad Software, La Jolla, California, USA). Survival curves were calculated using Kaplan-Meier estimate, and OS was measured from the date of enrollment up to 31 December 2018 or the time of death. Two-tailed Student's t-test or Mann-Whitney $\mathrm{U}$ test or two-way analysis of variance test with multiple group comparison adjustment (Dunnett's test) was used to analyze the statistical significance between groups. A $p$ value less than or equal to 0.05 was the threshold used to determine statistical significance.

\footnotetext{
Author affiliations

${ }^{1}$ Department of Melanoma, The University of Texas MD Anderson Cancer Center, Houston, Texas, USA
}

${ }^{2}$ Tianjin HengJia Biotechnology Development Co Ltd, Tianjin, China

${ }^{3}$ Mary Bird Perkins Cancer Center, Baton Rouge, Louisiana, USA

${ }^{4}$ Department of Oncology, Tianjin Beichen Hospital, Tianjin, China

${ }^{5}$ BostonGene Corporation, Waltham, Massachusetts, USA

${ }^{6}$ Department of Systems Biology, The University of Texas MD Anderson Cancer Center, Houston, Texas, USA

${ }^{7}$ Provincial Clinical College, Fujian Medical University, Fujian, China

${ }^{8}$ State Key Laboratory of Medicinal Chemical Biology, Nankai University, Tianjin, China

${ }^{9}$ Biological Sample Resource Sharing Center, Tianjin First Central Hospital, Tianjin, China

${ }^{10}$ Department of Immunology, Baylor College of Medicine, Houston, Texas, USA

${ }^{11}$ Department of Immunology, The University of Texas M.D. Anderson Cancer Center, Houston, Texas, USA

${ }^{12}$ Department of Biostatistics, The University of Texas MD Anderson Cancer Center, Houston, Texas, USA

Correction notice This paper has been updated to amend author affiliations.

Contributors FL, GL, and XD designed the clinical trial; FL and GL designed the peptide vaccines; FL and GL designed the experiments; FL and GL wrote and prepared the manuscript with feedback of coauthors PH, MD and CY; FL, LD, JR, AB, NK, VS, RA, NM, FF, CH, and GL analyzed the data; FL and WD conducted statistical plan; $X D, W F$, and $L H$ screened patients and performed the imaging, treatments, and biopsies; CC, QZ, LD, AHT, AK, KRJ, SDB, HS, YC, YW, MZ, DH, YZ, CY, PH, CY, and $Z W$ provided reagents or performed the experiments; $M S, S Z$, and $X D$ read and measured the patient CT scans. GL and XD provided project oversight.

Competing interests $\mathrm{CH}$ and $\mathrm{FL}$ are shareholders of Tianjin HengJia Biotechnology Development ('HengJia Biotech'). GL was a consultant for HengJia Neoantigen Biotechnology (Tianjin), a branch company of HengJia Biotech. LD, QZ, CC, and $\mathrm{CH}$ are employees of HengJia Biotech. MD is a consultant for Novartis, Roche/ Genentech, GSK, Array, Sanofi-Aventis, and Astrazeneca, and part of his research grants were from Roche/Genentech, GSK, Sanofi-Aventis, and Astrazeneca. United States patent applications have been filed on aspects of the described work, entitled: 'Immunogenic EGFR peptide compositions and their use in the treatment of cancer' (FL, GL), and 'Engineered T cell receptors targeting EGFR antigens and methods of use' (FL, GL). Chinese patent applications have been filed on aspects of the described work, entitled: 'The clinical application of specific T cell receptors based on EGFR-L858R mutation' (XD, LD, CH, and QZ), and 'Method for identifying tumor-specific T cell receptors' (XD). The remaining authors declare no competing financial interests.

Patient consent for publication Not required.

Ethics approval The study protocol conformed to the ethical guidelines of the 1975 Declaration of Helsinki and was approved by the internationally recognized Chinese Clinical Trial Registry (ChiCTR) and the Ethics Committee of Tianjin Beichen Hospital (ChiCTR No.: ChiCTR-IIR-16009867, weblink: http://www.chictr.org.cn/ showproj.aspx?proj=16736)

Provenance and peer review Not commissioned; externally peer reviewed.

Data availability statement Data are available in a public, open access repository. Data are available on reasonable request. Raw data of bulk RNA sequencing of cell lines, single cell TCR paired $\alpha \beta$ chain sequencing, TCR V $\beta C D R 3$ sequencing generated and analyzed during the current study are available through NCBI Sequence Read Archive (code SRP188005). DNA sequencing data (for finding mutations) with potential patient identification was not included in the informed consent of enrolled patients, but data without identifying information can be shared on reasonable request. All other data are also available from the corresponding author on request.

Supplemental material This content has been supplied by the author(s). It has not been vetted by BMJ Publishing Group Limited (BMJ) and may not have been peer-reviewed. Any opinions or recommendations discussed are solely those of the author(s) and are not endorsed by BMJ. BMJ disclaims all liability and responsibility arising from any reliance placed on the content. Where the content includes any translated material, BMJ does not warrant the accuracy and reliability of the translations (including but not limited to local regulations, clinical guidelines, terminology, drug names and drug dosages), and is not responsible for any error and/or omissions arising from translation and adaptation or otherwise.

Open access This is an open access article distributed in accordance with the Creative Commons Attribution Non Commercial (CC BY-NC 4.0) license, which permits others to distribute, remix, adapt, build upon this work non-commercially, 
and license their derivative works on different terms, provided the original work is properly cited, appropriate credit is given, any changes made indicated, and the use is non-commercial. See http://creativecommons.org/licenses/by-nc/4.0/.

\section{ORCID iDs}

Fenge Li http://orcid.org/0000-0003-3666-010X

Michael A Davies http://orcid.org/0000-0002-0977-0912

Chantale Bernatchez http://orcid.org/0000-0001-5926-3460

Patrick Hwu http://orcid.org/0000-0003-0554-2856

Gregory Lizee http://orcid.org/0000-0003-4449-7461

\section{REFERENCES}

1 Siegel RL, Miller KD, Jemal A. Cancer statistics, 2017. CA Cancer J Clin 2017;67:7-30.

2 Jemal A, Bray F, Center MM, et al. Global cancer statistics. CA Cancer J Clin 2011;61:69-90.

3 Nagano T, Tachihara M, Nishimura Y. Mechanism of resistance to epidermal growth factor receptor-tyrosine kinase inhibitors and a potential treatment strategy. Cells 2018;7:212.

4 Hastings K, Yu HA, Wei W, et al. EGFR mutation subtypes and response to immune checkpoint blockade treatment in non-smallcell lung cancer. Ann Oncol 2019;30:1311-20.

5 Yamada T, Hirai S, Katayama Y, et al. Retrospective efficacy analysis of immune checkpoint inhibitors in patients with EGFR-mutated nonsmall cell lung cancer. Cancer Med 2019;8:1521-9.

6 Bylicki O, Paleiron N, Margery J, et al. Targeting the PD-1/PD-L1 immune checkpoint in EGFR-mutated or ALK-translocated nonsmall-cell lung cancer. Target Oncol 2017;12:563-9.

7 liizumi S, Ohtake J, Murakami N, et al. Identification of novel HLA class II-restricted neoantigens derived from driver mutations. Cancers 2019;11:E266.

8 Chen F, Zou Z, Du J, et al. Neoantigen identification strategies enable personalized immunotherapy in refractory solid tumors. J Clin Invest 2019;129:2056-70.

9 Lu Y-C, Robbins PF. Cancer immunotherapy targeting neoantigens. Semin Immunol 2016;28:22-7.

10 Tran E, Turcotte S, Gros A, et al. Cancer immunotherapy based on mutation-specific CD4+ T cells in a patient with epithelial cancer. Science 2014;344:641-5.

11 Gupta RG, Li F, Roszik J, et al. Exploiting tumor neoantigens to target cancer evolution: current challenges and promising therapeutic approaches. Cancer Discov 2021;11:1024-39.

12 Carreno BM, Magrini V, Becker-Hapak M, et al. Cancer immunotherapy. A dendritic cell vaccine increases the breadth and diversity of melanoma neoantigen-specific T cells. Science 2015;348:803-8.

13 Kreiter S, Vormehr M, van de Roemer N, et al. Mutant MHC class II epitopes drive therapeutic immune responses to cancer. Nature 2015;520:692-6.

14 Sahin U, Derhovanessian E, Miller M, et al. Personalized RNA mutanome vaccines mobilize poly-specific therapeutic immunity against cancer. Nature 2017:547:222-6.

15 Li L, Goedegebuure SP, Gillanders WE. Preclinical and clinical development of neoantigen vaccines. Ann Oncol 2017;28:xii11-17.

16 Hailemichael Y, Dai Z, Jaffarzad N, et al. Persistent antigen at vaccination sites induces tumor-specific $C D 8^{+} \mathrm{T}$ cell sequestration, dysfunction and deletion. Nat Med 2013;19:465-72.

17 Ott PA, Hu Z, Keskin DB, et al. An immunogenic personal neoantigen vaccine for patients with melanoma. Nature 2017;547:217-21.

18 Keskin DB, Anandappa AJ, Sun J, et al. Neoantigen vaccine generates intratumoral T cell responses in phase Ib glioblastoma trial. Nature 2019;565:234-9.

19 Hilf N, Kuttruff-Coqui S, Frenzel K, et al. Actively personalized vaccination trial for newly diagnosed glioblastoma. Nature 2019;565:240-5.

$20 \mathrm{Li} \mathrm{F}$, Chen C, Ju T, et al. Rapid tumor regression in an Asian lung cancer patient following personalized neo-epitope peptide vaccination. Oncoimmunology 2016;5:e1238539.

21 Rizvi NA, Hellmann MD, Snyder A, et al. Cancer immunology. mutational landscape determines sensitivity to PD-1 blockade in non-small cell lung cancer. Science 2015;348:124-8.

22 Lawrence MS, Stojanov P, Polak P, et al. Mutational heterogeneity in cancer and the search for new cancer-associated genes. Nature 2013:499:214-8.

23 Hao J-J, Lin D-C, Dinh HQ, et al. Spatial intratumoral heterogeneity and temporal clonal evolution in esophageal squamous cell carcinoma. Nat Genet 2016;48:1500-7.

24 Levine AJ, Jenkins NA, Copeland NG. The roles of initiating truncal mutations in human cancers: the order of mutations and tumor cell type matters. Cancer Cell 2019;35:10-15.
25 Kobayashi Y, Mitsudomi T. Not all epidermal growth factor receptor mutations in lung cancer are created equal: perspectives for individualized treatment strategy. Cancer Sci 2016;107:1179-86.

26 Sidney J, Peters B, Frahm N, et al. HLA class I supertypes: a revised and updated classification. BMC Immunol 2008;9:1.

27 Harjanto S, Ng LFP, Tong JC. Clustering HLA class I superfamilies using structural interaction patterns. PLoS One 2014;9:e86655.

28 Jensen KK, Andreatta M, Marcatili P, et al. Improved methods for predicting peptide binding affinity to MHC class II molecules. Immunology 2018;154:394-406.

29 Becker K, Xu Y. Management of tyrosine kinase inhibitor resistance in lung cancer with EGFR mutation. World J Clin Oncol 2014;5:560-7.

30 Le X, Puri S, Negrao MV, et al. Landscape of EGFR-Dependent and -Independent Resistance Mechanisms to Osimertinib and Continuation Therapy Beyond Progression in EGFR-Mutant NSCLC. Clin Cancer Res 2018;24:6195-203.

31 Watanabe S, Hayashi $\mathrm{H}$, Haratani K, et al. Mutational activation of the epidermal growth factor receptor down-regulates major histocompatibility complex class I expression via the extracellular signal-regulated kinase in non-small cell lung cancer. Cancer Sci 2019;110:52-60.

32 Im JS, Herrmann AC, Bernatchez C, et al. Immune-modulation by epidermal growth factor receptor inhibitors: implication on anti-tumor immunity in lung cancer. PLOS One 2016;11:e0160004.

33 Venugopalan A, Lee M-J, Niu G, et al. EGFR-targeted therapy results in dramatic early lung tumor regression accompanied by imaging response and immune infiltration in EGFR mutant transgenic mouse models. Oncotarget 2016;7:54137-56.

34 Schoenborn JR, Wilson CB. Regulation of interferon-gamma during innate and adaptive immune responses. Adv Immunol 2007:96:41-101.

35 Schroder K, Hertzog PJ, Ravasi T, et al. Interferon-gamma: an overview of signals, mechanisms and functions. J Leukoc Biol 2004;75:163-89.

36 Chen N, Fang W, Zhan J, et al. Upregulation of PD-L1 by EGFR activation mediates the immune escape in EGFR-driven NSCLC: implication for optional immune targeted therapy for NSCLC patients with EGFR mutation. J Thorac Oncol 2015;10:910-23.

37 Lin K, Cheng J, Yang T, et al. EGFR-TKI down-regulates PD-L1 in EGFR mutant NSCLC through inhibiting NF- $\mathrm{KB}$. Biochem Biophys Res Commun 2015:463:95-101.

38 González-Galarza FF, Takeshita LYC, Santos EJM, et al. Allele frequency net 2015 update: new features for HLA epitopes, KIR and disease and HLA adverse drug reaction associations. Nucleic Acids Res 2015;43:D784-8.

39 Kobayashi S, Boggon TJ, Dayaram T, et al. EGFR mutation and resistance of non-small-cell lung cancer to gefitinib. N Engl J Med 2005;352:786-92.

$40 \mathrm{Xu} \mathrm{J}$, Wang J, Zhang S. Mechanisms of resistance to irreversible epidermal growth factor receptor tyrosine kinase inhibitors and therapeutic strategies in non-small cell lung cancer. Oncotarget 2017:8:90557-78.

41 El Kadi N, Wang L, Davis A, et al. The EGFR T790M mutation is acquired through AICDA-mediated deamination of 5-methylcytosine following TKI treatment in lung cancer. Cancer Res 2018;78:6728-35.

42 McGranahan N, Rosenthal R, Hiley CT, et al. Allele-specific HLA loss and immune escape in lung cancer evolution. Cell 2017;171:1259-71.

43 Bedognetti Det al. Society for immunotherapy of cancer (SITC) cancer immune responsiveness Task force and working groups, toward a comprehensive view of cancer immune responsiveness: a synopsis from the SITC workshop. J Immunother Cancer 2019;7:131.

44 Rosenthal R, Cadieux EL, Salgado R, et al. Neoantigen-directed immune escape in lung cancer evolution. Nature 2019;567:479-85.

45 Overwijk WW. Human CD4(+) T cells spontaneously detect somatic mutations in cancer cells. Nat Med 2015;21:12-14.

46 Malekzadeh P, Pasetto A, Robbins PF, et al. Neoantigen screening identifies broad TP53 mutant immunogenicity in patients with epithelial cancers. J Clin Invest 2019;129:1109-14.

47 Pol J, Bloy N, Buqué A, et al. Trial Watch: Peptide-based anticancer vaccines. Oncoimmunology 2015;4:e974411.

48 Goodridge JP, Lee N, Burian A, et al. HLA-F and MHC-I open conformers cooperate in a MHC-I antigen cross-presentation pathway. J Immunol 2013;191:1567-77.

49 Yamaoka T, Ohba M, Ohmori T. Molecular-targeted therapies for epidermal growth factor receptor and its resistance mechanisms. Int J Mol Sci 2017;18:E2420.

50 Wirth TC, Kühnel F. Neoantigen targeting-dawn of a new era in cancer immunotherapy? Front Immunol 2017;8:1848.

51 Lu Y-C, Robbins PF. Cancer immunotherapy targeting neoantigens. Semin Immunol 2016;28:22-7. 
52 Andreatta M, Nielsen M. Gapped sequence alignment using artificial neural networks: application to the MHC class I system. Bioinformatics 2016;32:511-7.

53 Tumeh PC, Harview CL, Yearley JH, et al. PD-1 blockade induces responses by inhibiting adaptive immune resistance. Nature 2014;515:568-71.

54 Cohen CJ, Li YF, El-Gamil M, et al. Enhanced antitumor activity of T cells engineered to express T-cell receptors with a second disulfide bond. Cancer Res 2007;67:3898-903.

55 Wingett SW, Andrews S. FastQ screen: a tool for multi-genome mapping and quality control. F1000Res 2018;7:1338.

56 Bray NL, Pimentel H, Melsted P, et al. Near-optimal probabilistic RNA-seq quantification. Nat Biotechnol 2016;34:525-7.
57 Conesa A, Madrigal P, Tarazona S, et al. A survey of best practices for RNA-Seq data analysis. Genome Biol 2016;17:13.

58 Schubert M, Klinger B, Klünemann M, et al. Perturbation-response genes reveal signaling footprints in cancer gene expression. Nat Commun 2018;9:20.

59 Subramanian A, Tamayo P, Mootha VK, et al. Gene set enrichment analysis: a knowledge-based approach for interpreting genome-wide expression profiles. Proc Natl Acad Sci U S A 2005;102:15545-50.

60 Liang C, Li Y, Luo J. A novel method to detect functional microRNA regulatory modules by bicliques merging. IEEE/ACM Trans Comput Biol Bioinform 2016;13:549-56. 


\section{Correction: Neoantigen vaccination induces clinical and} immunologic responses in non-small cell lung cancer patients harboring EGFR mutations

Li F, Deng L, Jackson KR, et al. Neoantigen vaccination induces clinical and immunologic responses in non-small cell lung cancer patients harboring EGFR mutations. J Immuno Ther Cancer 2021;9:e002531. doi: 10.1136/jitc-2021-002531

This paper has been updated to amend author affiliations.

(C) Author(s) (or their employer(s)) 2021. No commercial re-use. See rights and permissions. Published by BMJ.

J Immunother Cancer 2021;9:e002531corr1. doi:10.1136/jitc-2021-002531corr1

(A) Check for updates 